\title{
Biothermodynamics of live cells: a tool for biotechnology and biochemical engineering
}

\author{
Urs von Stockar \\ Communicated by J. M. Rubi, Barcelona, Spain, and \\ J. U. Keller, Siegen, Germany
}

\begin{abstract}
The aim of this contribution is to review the application of thermodynamics to live cultures of microbial and other cells and to explore to what extent this may be put to practical use. A major focus is on energy dissipation effects in industrially relevant cultures, both in terms of heat and Gibbs energy dissipation. The experimental techniques for calorimetric measurements in live cultures are reviewed and their use for monitoring and control is discussed. A detailed analysis of the dissipation of Gibbs energy in chemotrophic growth shows that it reflects the entropy production by metabolic processes in the cells and thus also the driving force for growth and metabolism. By splitting metabolism conceptually up into catabolism and biosynthesis, it can be shown that this driving force decreases as the growth yield increases. This relationship is demonstrated by using experimental measurements on a variety of microbial strains. On the basis of these data, several literature correlations were tested as tools for biomass yield prediction. The prediction of other culture performance characteristics, including product yields for biorefinery planning, energy yields for biofuel manufacture, maximum growth rates, maintenance requirements, and threshold concentrations is also briefly reviewed.
\end{abstract}

\section{Introduction}

Thermodynamics has been an essential scientific fundament of chemical sciences and chemical engineering for a very long time. It has been of in- 
valuable help in a multitude of tasks, including judging whether reactions are feasible and under which physical-chemical conditions, estimating energy requirements and dissipation rates, assessing the safety of chemical operations, and quantifying equipment design at laboratory and industrial scales. Thermodynamic analysis thus plays an absolutely central role in chemistry, chemical engineering, and chemical process development.

In biotechnology, however, the development of thermodynamic analysis has lagged behind the application of the other two fundamental concepts underlying most quantitative theories and calculations in engineering sciences, these being balances (e.g., mass, elemental, energy, momentum) and kinetics (e.g., momentum and mass transfer, reaction and growth kinetics). This is probably not surprising in view of the daunting complexity of the biological world, characterized by giant biological molecules, multiple driving forces, heterogeneity of phases, and a multitude of biological, chemical, and physical processes occurring under rigorously irreversible conditions [1]. The resulting lack of basic data concerning biomolecular properties, thermodynamic equilibrium position, formulation of driving forces, and energy efficiency relations in biotechnology is one among several reasons why the development and design of biotechnological processes is still today in many cases carried out in an essentially empirical fashion and why bioprocesses often are not as thoroughly optimized as many chemical processes.

Yet one would expect that in biochemical engineering, too, thermodynamic analysis should be able to predict whether growth of a given type of cells or a specified type of metabolic reactions is feasible and under what conditions. On the basis of such analyses it ought to be possible to roughly estimate key parameters of biotechnological cultures and thus to address the economic viability of the process before even performing experiments. Once the first measurements are carried out, the thermodynamic predictions could be used as benchmarks, in the sense that experimental results staying far behind the calculations might indicate that there may be ample room for improvement. All of this would be of invaluable help not only in bioprocess development, but also in biotechnological operations at the industrial scale and in scientific experimentation in general.

Fortunately, an increasing awareness of this potential during recent years, as well as newly available data, has resulted in a growing number of scientific papers dealing with what is becoming known as biochemical thermodynamics or biothermodynamics [2,3]. Judging from this literature, biothermodynamics may today be subdivided into three large fields according to the scale of the system that is thermodynamically described (Figure 1). 


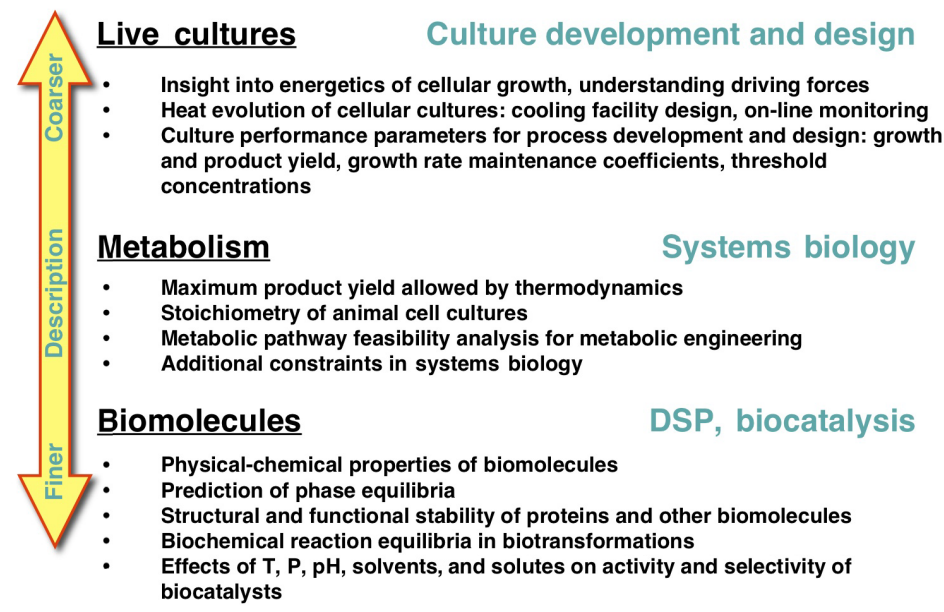

Figure 1. Classification of developments in biothermodynamics according to level of system description [4].

The coarsest level of description is an application of thermodynamics directly to whole live cultures. Although only rarely used in practice, application of thermodynamic concepts to live cultures could be of great help in culture design, development, and operation.

The next lower level of description is an application of thermodynamics to the whole or to part of the metabolism. It clearly has the potential to contribute to the construction of genome-wide mathematical models of the metabolism and was therefore developed in the framework of systems biology. This area is, however, the most recent and thus not as well developed as the other two. Both the application to metabolism and to live cells concerns functioning systems operating far from equilibrium.

The most fundamental level of description is molecular biothermodynamics. As in chemical technology, this field may have an enormous impact on the design and development of downstream processing, and in addition also on biocatalysis. It is by far the best developed of the three.

The aim of this contribution is to review the status of the first of these three areas and to explore to what extent the respective thermodynamic concepts may be put to practical use for industrially relevant cultures. It is planned to address biothermodynamics of the metabolism in a future review, but in view of the relatively better state of matters in molecular biothermodynamics and because non-equilibrium thermodynamics is of less use in this area than in the first two, a review of this topic is not foreseen in the present environment. 
After a short formal treatment of the application of the First Law of Thermodynamics to live cell cultures, the quantitative measurement of heat generation by cellular cultures will be treated. On the basis of the Second Law of Thermodynamics, the formulation of entropy and Gibbs energy balances for cellular cultures will then briefly be addressed. Experimental data on Gibbs energy dissipation in growing microbial cell cultures will be discussed for a whole series of different cases and compared with measured heat dissipation data. This will be used to gain insight into the nature of the driving forces for microbial growth and as a basis for reviewing concepts that are able to predict important culture performance parameters. The biomass yield will serve as the main example for illustrating the prediction of such parameters, but product yields, growth rate, maintenance requirements, and heat evolution rates will also be briefly addressed.

\section{Fundamentals of biothermodynamics}

\subsection{The First Law: Heat generation in live cultures and biocalorimetry}

\subsubsection{Why should we deal with heat dissipation rates?}

Thermodynamics literally means "the force of heat." The thermodynamics of cellular growth is thus clearly intimately linked to the measurable phenomenon of heat dissipation. Understanding the thermodynamics of the growth of microbial or other cells thus implies studying, among other things, why growing cellular cultures generate heat and how much.

Indeed, heat is a very universal byproduct of all biological phenomena, including those that are exploited in biotechnology at large scale (Table 1). Yet heat effects in cellular cultures often go unnoticed when one is working with conventional laboratory equipment because most of the heat released by the culture is lost to the environment too quickly to give rise to a perceivable temperature increase. This, however, is completely different in microbial cultures at large scale [5-8]. As opposed to laboratory reactors, industrial-size fermenters operate nearly adiabatically due to their much smaller surface-to-volume ratio. Thus all the heat released by the culture must be removed by appropriate cooling facilities. It is therefore of great practical importance to have sufficient quantitative information on microbial heat release when designing the cooling facilities for biotechnological processes. 
Table 1. Heat generation rates in biological processes.

\begin{tabular}{|c|c|c|c|c|}
\hline \multirow[t]{2}{*}{ Organism } & \multirow[t]{2}{*}{ Reference } & \multicolumn{3}{|c|}{ Heat generation } \\
\hline & & $\begin{array}{l}\text { Watts per } \\
\text { organism }\end{array}$ & $\begin{array}{l}\text { Watts } \\
\text { per } \\
\text { kg live } \\
\text { mass }\end{array}$ & $\begin{array}{l}\text { Watts } \\
\text { per } \\
\text { kg dry } \\
\text { mass }\end{array}$ \\
\hline Man & Frenz [78] & 140 & 1.7 & - \\
\hline Guinea pig & $\begin{array}{l}\text { Lavoisier and } \\
\text { de Laplace [79] }\end{array}$ & 3.5 & 3 & - \\
\hline $\begin{array}{l}\text { Lotus flower } \\
\text { (during } \\
\text { thermogenesis } \\
\text { period) }\end{array}$ & $\begin{array}{l}\text { Lamprecht } \\
\text { et al. [80] }\end{array}$ & 0.3 & 6 & - \\
\hline $\begin{array}{l}\text { Bumble bee in } \\
\text { nest (June) }\end{array}$ & $\begin{array}{l}\text { Schultze- } \\
\text { Motel [81] }\end{array}$ & 0.005 & 30 & - \\
\hline $\begin{array}{l}\text { Bumble bee in } \\
\text { nest (July) }\end{array}$ & $\begin{array}{l}\text { Schultze- } \\
\text { Motel [81] }\end{array}$ & 0.03 & 200 & - \\
\hline $\begin{array}{l}\text { Yeast culture } \\
\text { at rest }\end{array}$ & $\begin{array}{l}\text { Birou and von } \\
\text { Stockar [82] }\end{array}$ & - & 5 & 25 \\
\hline $\begin{array}{l}\text { Yeast culture } \\
\text { growing at } \\
\mu_{\max }\end{array}$ & $\begin{array}{l}\text { Birou and von } \\
\text { Stockar [82] }\end{array}$ & - & 250 & 1250 \\
\hline $\begin{array}{l}\text { Methanogenic } \\
\text { bacteria } \\
\text { growing at } \\
\mu_{\max }\end{array}$ & Schill et al. [51] & - & 900 & 4500 \\
\hline
\end{tabular}

The continuous generation of heat by microbial cultures could also be used as a basis for an on-line monitoring of the microbial activity and metabolism. If the temperature increase in the cooling water, its flow rate, and the other relevant energy exchange terms such as agitation and evaporation rates were measured systematically, the heat dissipation rate of the cellular culture could quantitatively be monitored on-line in industrial fermenters. The information contained in this signal could, in principle, be used together with other on-line data in order to optimize the bioprocess and for on-line process control [8]. 


\subsubsection{The First Law and energy balances}

Heat generation rates are usually measured in some sort of calorimeters or suitably equipped laboratory reactors on the basis of an energy balance. Such devices are often dynamically operated open systems as the one depicted in Figure 2. Applying the First Law to such systems yields

$$
\rho V \cdot \bar{c}_{\mathrm{P}} \cdot \frac{\mathrm{d} T}{\mathrm{~d} t}=\dot{Q}+\dot{W}+\sum_{e} \dot{V}_{e} \rho \bar{c}_{\mathrm{P}} \cdot\left(T_{e}-T\right)-\sum_{j} \Delta_{r_{j}} H \cdot r_{j} V,
$$

where $\dot{V}_{e}$ and $T_{e}$ denote the volumetric flow rate and the temperature, respectively, of the material flowing into the system through the $e$-th exchange port, whereas $\Delta_{r j} H$ indicates the molar enthalpy of the $j$-th reaction occurring inside. The derivation of Eq. (1) from the First Law of Thermodynamics is given in Appendix A (see also von Stockar et al. [9]). Equation (1) is the classical basis for relating measured heat release rates $\dot{Q}$ to the processes occurring in reaction calorimeters. It allows for several chemical transformations, each characterized by its $\Delta_{r j} \mathrm{H}$ value.

In biological calorimetry, one is often not interested in a multitude of the chemical transformations as suggested by the last term of Eq. (1), but only in the cellular growth reaction proceeding at rate $r_{X}$ and characterized by $\Delta_{r} H_{X}$. In simple cases, such as in microbial growth, this growth reaction may be formulated as a so-called macrochemical equation with fixed

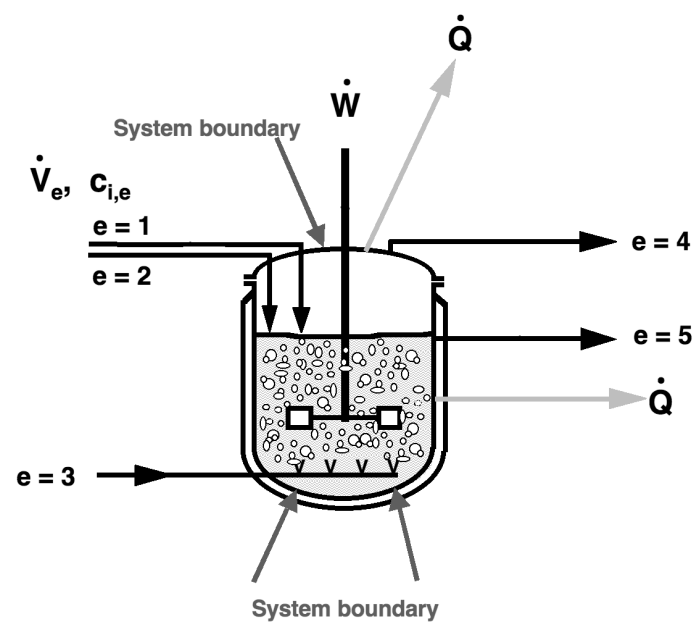

Figure 2. System considered for establishing the energy balance Eq. (1) for heat measurements. 
stoichiometry. A typical example reads:

$$
\frac{1}{Y_{\mathrm{X} / \mathrm{S}}} \mathrm{S}+Y_{\mathrm{A} / \mathrm{X}} \mathrm{A}+Y_{\mathrm{N} / \mathrm{X}} \mathrm{NH}_{3} \rightarrow \mathrm{X}+Y_{\mathrm{P} / \mathrm{X}} \mathrm{P}+Y_{\mathrm{C} / \mathrm{X}} \mathrm{CO}_{2}+Y_{\mathrm{W} / \mathrm{X}} \mathrm{H}_{2} \mathrm{O},
$$

where $\mathrm{S}, \mathrm{A}, \mathrm{X}$, and $\mathrm{P}$ represent the carbon and energy source, an electron acceptor, the newly grown biomass, and a catabolic waste product, respectively. $Y_{\mathrm{X} / \mathrm{S}}$ denotes the biomass yield on the carbon and energy source (C-mol of biomass / C-mol of S), $Y_{i / \mathrm{X}}$ the other yields. All these yields may be regarded as stoichiometric coefficients of the growth reaction. An experimentally observed example of Eq. (2) is presented in Appendix B.

It is then assumed that all other chemical transformations such as neutralization, gassing out, etc., only contribute constant minor signals and may be "lumped" into the base line. The same is done with heat losses that cannot be picked up by the heat measurement system. Hence the equation is thus often presented in a simplified form as follows:

$$
\rho V \cdot \bar{c}_{\mathrm{P}} \cdot \frac{\mathrm{d} T}{\mathrm{~d} t}=\dot{Q}+\dot{W}+\sum_{e} \dot{V}_{e} \rho \bar{c}_{\mathrm{P} e}\left(T_{e}-T\right)-\Delta_{r} H_{\mathrm{X}} \cdot r_{\mathrm{X}} V,
$$

where $\dot{Q}$ now represents the heat signal deviating from this base line only. $\dot{V}_{e}$ and $\overline{\mathrm{c}}_{\mathrm{Pe}}$ stand for the entering molar flow rate and the mean heat capacity of the mixture entering through the $e$-th exchange port, respectively, and $\Delta_{r} H_{\mathrm{X}}$ represents the heat of reaction (2), and $r_{\mathrm{X}}$ denotes its rate (C-mol $\left.\mathrm{m}^{-3} \mathrm{~s}^{-1}\right)$.

On the basis of Eq. (3), it is possible to (i) predict heat dissipation rates for live cultures, or conversely to (ii) use measured heat dissipation rates to determine the enthalpy of growth, $\Delta_{r} H_{\mathrm{X}}$, or to (iii) observe the biological activity, i.e., $r_{\mathrm{X}}$, on-line. In order to measure heat dissipation $\dot{Q}$, a calorimetric technique has to be applied to the bioreactor.

\subsubsection{Calorimetric measuring techniques}

In order to obtain results meaningful for biotechnology, the calorimetric experiment must be performed under tightly controlled culture conditions. Moreover, all other relevant process parameters and variables must be measured during the culture. Conventional microcalorimeters do not normally meet these criteria, although microcalorimeter designs exist that afford at least some mixing and oxygenation, so that aerobic cultures with modest oxygen uptakes can be studied (cf. e.g., [10]). Yet experimental problems remain severe for the highly aerobic cultures of interest in biotechnology. 


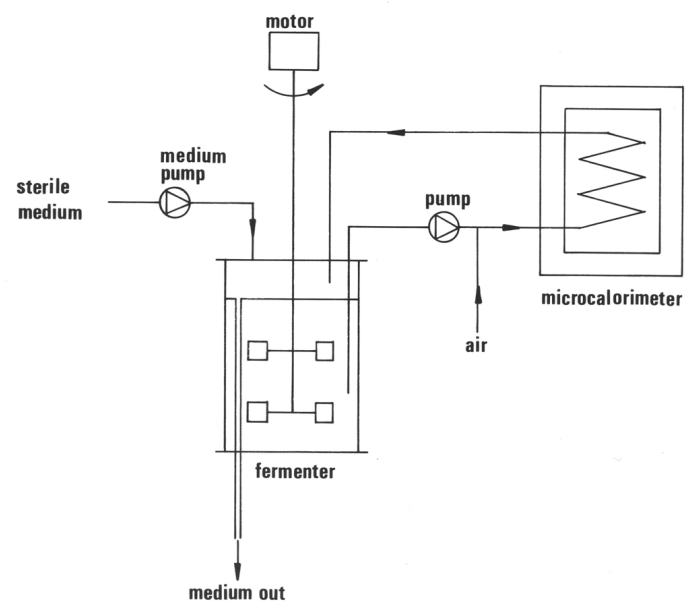

Figure 3. Principles of flow-through calorimeters. Reprinted with permission from von Stockar and Marison [5].

As a result, a number of researchers tried to alleviate problems associated with microcalorimeters by growing the culture in a standard benchscale fermenter and by pumping a continuous sample stream through a flow microcalorimeter (Figure 3). Experience with this calorimetric technique has been reported, e.g., by Gustafsson and co-workers [11,12], who studied the energy balance during growth of the yeast Saccharomyces cerevisiae for different periods during batch growth, and by Guan et al. [13], Guan and Kemp [14], Kemp and Guan [15], who demonstrated the usefulness of this approach in measuring the feeble heat dissipation rates of animal cell cultures. More recently, Maskow et al. [16] used this approach for controlling the conversion of toxic substrates by bacteria.

Other efforts over the last three decades have resulted in different types of calorimeters at the bench scale. These can be operated like standard laboratory fermenters but enable measurement of the heat dissipation rate in situ. Consequently, they are especially well suited for quantitative research under process-relevant conditions and for the highly aerobic cultures of interest in biotechnology and biochemical engineering. The first development of this kind was due to Cooney et al. [17] and became known as "dynamic calorimetry". Meanwhile several research groups have operated bench-scale calorimeters of various types [5, 18-26]. Figure 4 shows one of these, based on the measuring principle known as "isothermal reaction calorimetry". A commercial version called RC-1 is marketed for chemical 


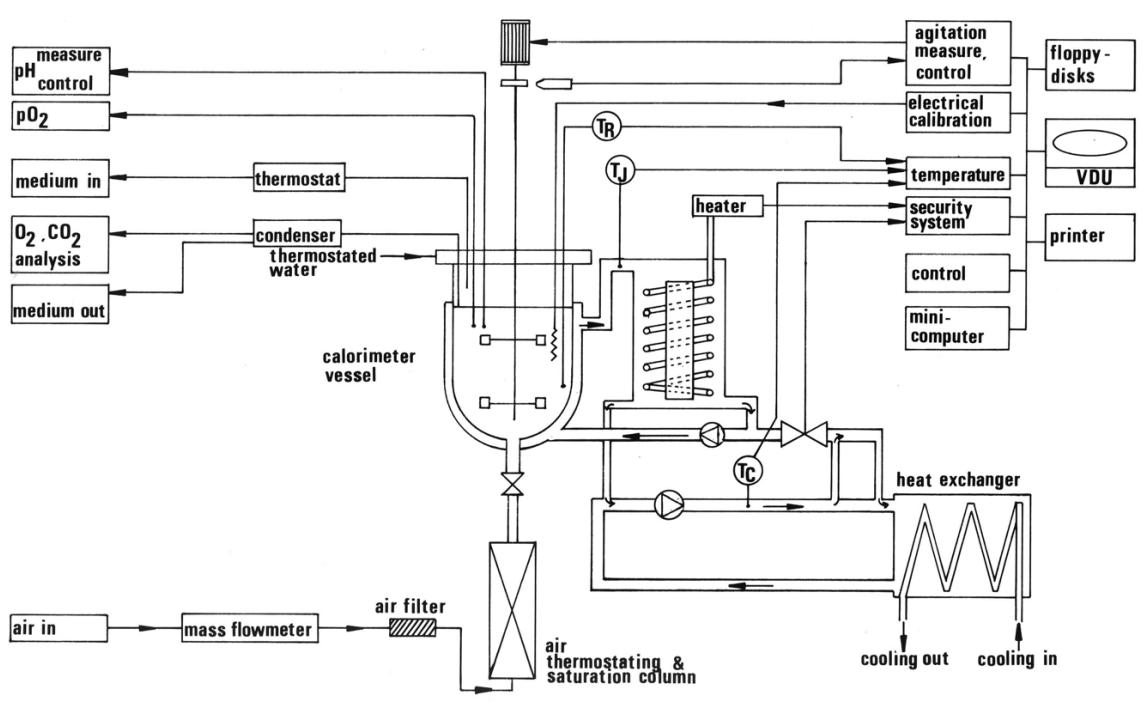

Figure 4. Principles of isothermal reaction calorimetry (reprinted with permission from von Stockar and Marison [5]).

reactions by Mettler AG, Greifensee, Switzerland. It can be transformed into a bioreactor [27-29].

The RC-1 is composed of a 21 jacketed glass reactor, through the jacket of which is pumped a silicone oil at the rate of $21 \mathrm{~s}^{-1}$ (see Figure 4). The temperature of this oil $T_{\mathrm{J}}$ is controlled by a special thermostat in the circulatory system and can be modified quite rapidly. This is achieved by mixing warm and cool oil in different proportions using an electronic valve operated automatically by a computer. The temperature $T_{\mathrm{R}}$ in the reaction vessel itself is measured very accurately and the control algorithm adapts the temperature $T_{\mathrm{J}}$ continuously in such a way that $T_{\mathrm{R}}$ remains at its set point value. The different $T_{\mathrm{R}}-T_{\mathrm{J}}$ is a measure for the heat flow rate transferred to the jacket (W) and may be used to determine the latter according to Eq. (4):

$$
-\dot{Q}=U A\left(T_{\mathrm{R}}-T_{\mathrm{J}}\right),
$$

where $U$ and $A$ stand for the heat transmission coefficient $\left(\mathrm{W} \mathrm{m}^{-2} \mathrm{~K}^{-1}\right.$ ) and the effective heat transfer area $\left(\mathrm{m}^{2}\right)$, respectively. The product $U \cdot A$ can be measured by activating an internal calibration heater.

Bench-scale calorimeters cannot normally compete with microcalorimeters with respect to measurement sensitivity. Whereas the latter can monitor heat signals as low as $10 \mu \mathrm{W}$ per ml of sample volume, the detection 


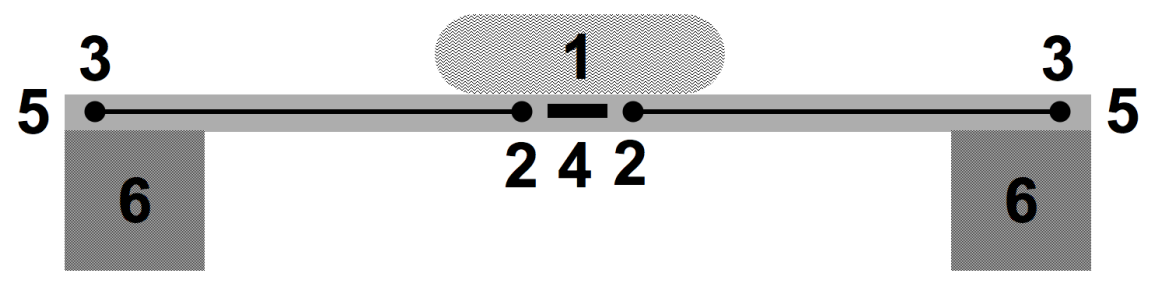

Figure 5. Structure of a chip calorimeter. Components: (1) sample, (2) hot junctions, (3) cold junctions, (4) chip heater, (5) extremely thin membrane, (6) rim. Adapted from Maskow and Harms [31]. The sample (1) could be as small as a few dozens of nanoliters [33].

limit of the commercial version of the RC-1 is about $100 \mathrm{~mW} / 1$. Due to systematic improvements of the Bio RC-1, it is possible to push this limit down to $\pm 5-10 \mathrm{~mW} / 1$, which comes at least close to the performance of microcalorimeters [30].

In contrast to the modified RC-1, the calorimeters designed and operated by van Kleeff et al. [19], Meier-Schneiders [23], and Maskow and Babel [26] are based on heat balances around the fermenter vessel. Such calorimeter-fermenters are thermally insulated from their environment to minimize heat losses. An active compensation heater/cooler is installed in the fermentation broth in order to keep $T_{\mathrm{R}}$ at the set point. By performing a heat balance incorporating all known heat exchange effects with the environment, the heat generation due to the biological activity $\left(\Delta_{r} H_{\mathrm{X}} \cdot r_{\mathrm{X}} V\right)$ can be calculated based on Eq. (3).

In more recent times it has been demonstrated that the problem associated with biotechnology cannot only be alleviated by scaling the instrument up to the bench scale, but rather by scaling it down to yield a chip calorimeter [31-34]. The principle of this device, shown in Figure 5, consists in measuring either the adiabatic temperature increase that results from a reacting mixture in a sample injected into the measuring cell, or the steadystate temperature during continuous flow of sample as compared with the temperature obtained from a continuous flow of reference sample. The temperature sensors, the heat sink, and the sample channels are integrated into a single chip. Due to the miniaturization the measurement is extremely fast and heat losses are essentially minimized. For the injection mode the signal-to-noise ratio is therefore virtually independent of the sample volume, thus permitting the investigation of very small samples. The chip 
calorimeter is believed to hold considerable potential in monitoring bioreactors in the at-line mode, in a flow-through mode, and also in calorimetric investigations of biofilms.

\subsubsection{Typical heat generation curves during microbial growth}

A typical heat release curve obtained during the aerobic growth of a yeast culture is shown in Figure 6, [35]. As can be seen from the figure, the heat dissipation rate $\dot{q}$ as a function of time may be integrated to obtain a curve showing the total heat $(Q)$ generated up to a certain point in time, which parallels the biomass concentration $x$ ( $\mathrm{g}^{-1}$ dry mass) quite nicely. It is therefore not surprising to find a straight correlation when plotting $Q$ as a function of $x$ (Figure 7, left). The slope of this correlation indicates the amount of heat generated per unit dry biomass formed and is a measure for the molar enthalpy of reaction $\Delta_{r} H_{\mathrm{X}}$ as shown in the next paragraph.

Equation (3) may be simplified for such measurements if (i) the calorimeter is operated strictly isothermally, thus eliminating its left-hand side, (ii) the effect of mechanical work done on the culture such as mechanical stirring may be assumed to be small and constant, thus allowing to handle $\dot{W}$ in the base line, and (iii) the calorimeter is either a closed system or if entering streams are carefully pre-thermostated such that $T_{e}=T$, therefore also doing away with the second last right-hand side term. Equation (3) then reduces to

$$
\begin{aligned}
\dot{Q} & =\Delta_{r} H_{\mathrm{X}} \cdot r_{\mathrm{X}} V, \quad \text { or } \\
\frac{\dot{Q}}{r_{\mathrm{X}} V} & =\frac{\dot{q}}{r_{\mathrm{X}}} \approx \frac{Q}{x-x_{0}}=\Delta_{r} H_{\mathrm{X}} .
\end{aligned}
$$

The negative of this value is sometimes called the "heat yield" $\left(\mathrm{Y}_{Q / \mathrm{X}}\right)$.

As can be seen from Figure 7 (right), the cumulative amount of heat released also correlates linearly with the total amount of oxygen consumed, thereby giving rise to another type of heat yield $\left(Y_{Q / \mathrm{O}}\right)$ or the molar enthalpy of reaction per mole of oxygen consumed $\left(\Delta_{r} H_{\mathrm{O}}\right)$. Similar linear correlations would be found with the amounts of any of the major metabolites consumed or released appearing in Reaction (2), provided the stoichiometry of this reaction stays constant during growth. The different corresponding molar enthalpies of reaction $\Delta_{r} H_{i}$ are linked to each other by the stoichiometry, Eq. (2). It may be shown that for the example of Reaction (2) measuring one of the stoichiometric coefficients and one of these reaction enthalpies suffices for determining all the other coefficients. Con- 


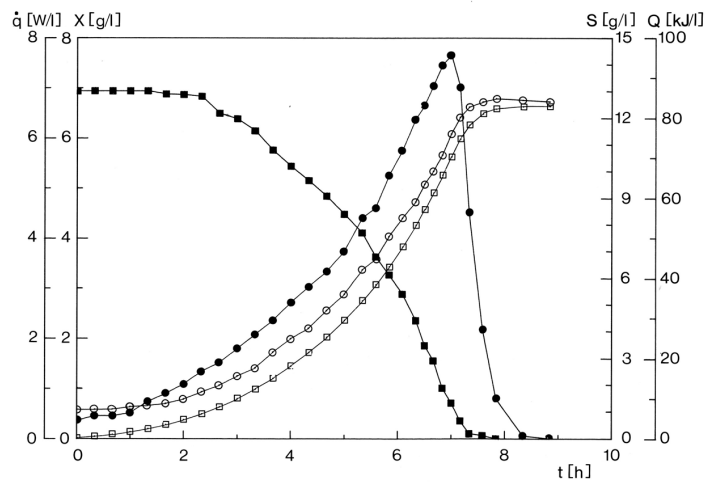

Figure 6. Heat release during batch growth of yeast Kluyveromyces marxianus. Solid squares: substrate concentration $s(\mathrm{~g} / \mathrm{l})$; solid dots: heat evolution rate $\dot{q}(\mathrm{~W} / \mathrm{l})$; open squares: dry biomass grown $x-x_{0}(\mathrm{~g} / \mathrm{l})$; open circles: integrated heat $Q(\mathrm{~kJ} / \mathrm{l})$. Reprinted with permission from Birou et al. [35].
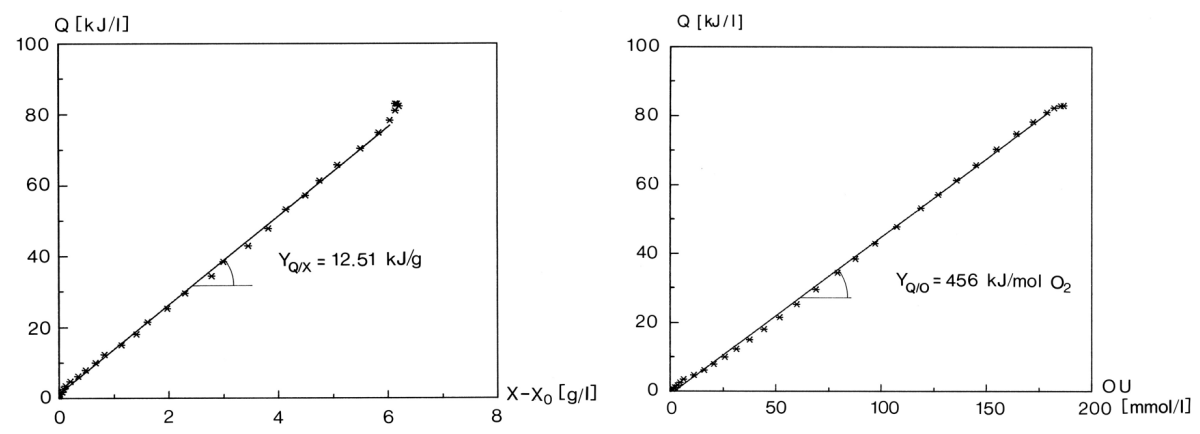

Figure 7. Correlation of total heat released with amount of dry biomass grown (left) and with total amount of oxygen consumed (right) [35].

versely, the enthalpies of reaction $\Delta_{r} H_{i}$ may be calculated if two stoichiometric coefficients in Eq. (2) are known.

A selection of experimentally measured heat yields for aerobic and anaerobic growth is reported in Table 2. In this table the energy content of the substrates is indicated by the degree of reduction. As explained below, the degree of reduction closely parallels the energy content of a substance in term of its heat of combustion, $\Delta_{\mathrm{c}} H_{i}$.

It may be seen that the biomass yield $Y_{\mathrm{X} / \mathrm{S}}$ increases with the degree of reduction, whereas the heat yield $Y_{Q / \mathrm{X}}$ appears to stay more or less constant for low degrees of reduction but to increase at high $\gamma_{S}$. On the other hand, the heat yield per oxygen $Y_{Q / O}$ remains fairly constant, scattering 


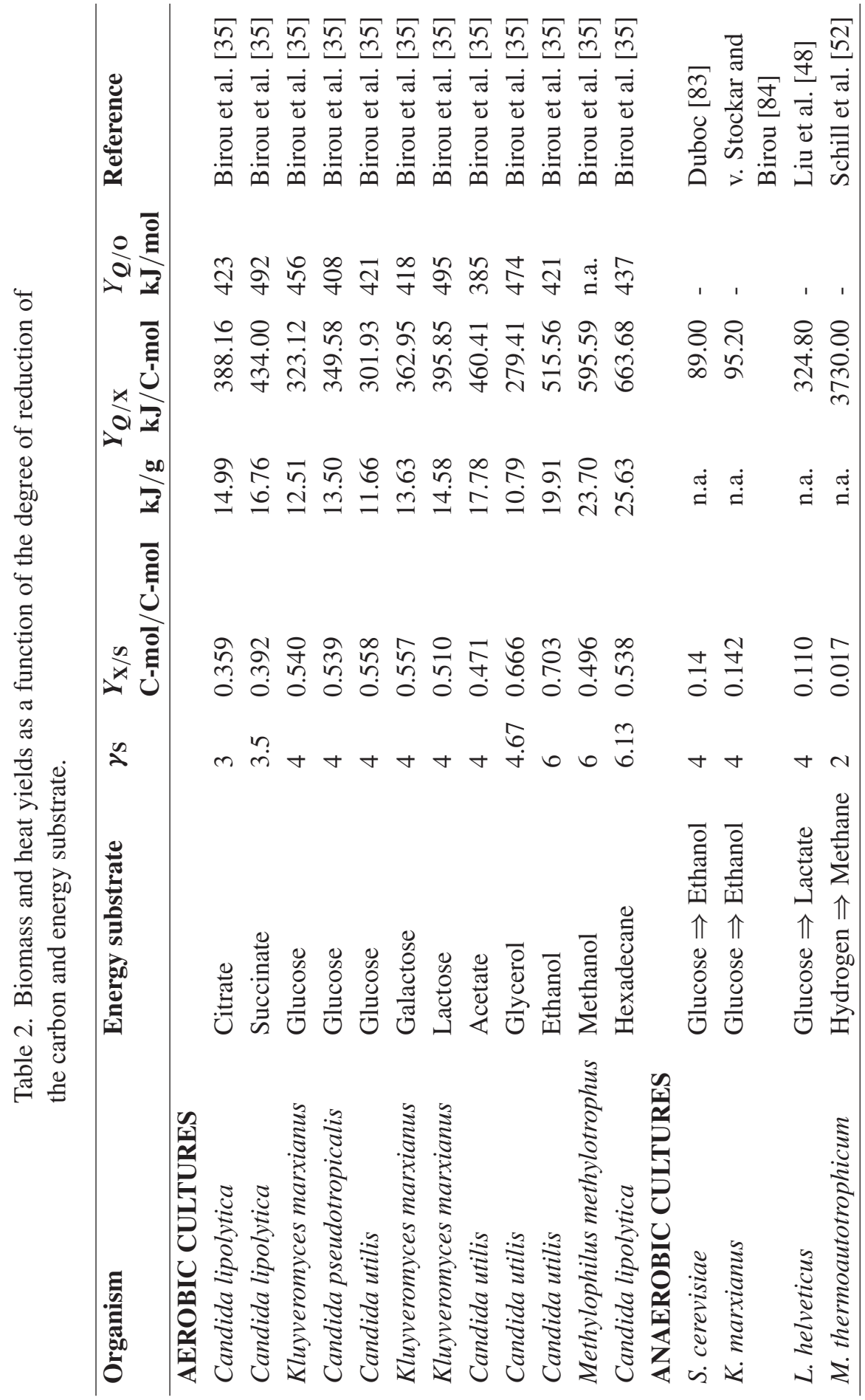


around a value of $450 \mathrm{~kJ} \mathrm{~mol}^{-1}$, despite considerable variations of the heat per biomass grown $Y_{Q / \mathrm{X}}$, and of the growth yield itself [35].

The latter observation may be explained by the fact that the energy content of any organic compound in terms of its heat of combustion is directly proportional to the amount of $\mathrm{O}_{2}$ consumed upon combustion. On the other hand, the degree of reduction indicates by definition the number of electrons transferred to oxygen by the combustion of a $\mathrm{C}$-mole of a given organic compound, which is proportional to the number of mole of oxygen consumed. Therefore, the heat of combustion of any compound is also directly proportional to its degree of reduction. The proportionality coefficient is very nearly the same for most organic compounds [36-38]. Thus the standard C-molar enthalpy of combustion $\Delta_{\mathrm{c}} H_{i}^{0}$ may be estimated quite accurately as

$$
\Delta_{\mathrm{c}} H_{i}^{0}=Q_{0} \cdot \gamma_{i}
$$

where $Q_{0}$ was shown experimentally to amount to about $-109.0 \mathrm{~kJ} /$ degree of reduction [39].

It is obvious that Eq. (7) predicts that the enthalpies of combustion are different for organic compounds with different degrees of reduction. A Cmole of ethanol, for example, will exhibit a heat of combustion $150 \%$ of that of a C-mole of a common carbohydrate, because the degrees of reduction of ethanol and carbohydrate are 6 and 4, respectively. However, if $\Delta_{\mathrm{c}} H_{i}^{0}$ is expressed per 1 mole of oxygen consumed, the same value of $+440 \mathrm{~kJ}$ per mole of $\mathrm{O}_{2}$ is predicted because, irrespective of the nature of the electron donor, oxygen has always the same degree of reduction of $\gamma_{\mathrm{O}_{2}}=-4$.

Since growth, too, is a partial combustion, the same ratio of heat generation to oxygen consumption must hold. As a result, the ratio of heat production to oxygen consumption in all aerobic processes, whatever the strain involved and even if some amounts of fermentation products are formed, is always approximately given by $4 Q_{0}$, i.e., $440 \mathrm{~kJ} / \mathrm{mol}$. This is in fair agreement with the observed average value of $450 \mathrm{~kJ} \mathrm{~mol}^{-1}$ [35]. This ratio, called sometimes the calo-respirometric coefficient, is the basis for the so-called "indirect calorimetry."

For anaerobic growth the above argument would predict an athermal process since no oxygen is consumed. However, as reported in Table 2 the enthalpy change per C-mole of biomass grown may be smaller or even considerably larger than for aerobic growth. While in aerobic growth the dependence of $Y_{\mathrm{X} / \mathrm{S}}$ and $Y_{Q / \mathrm{S}}$ on the degree of reduction $\gamma_{\mathrm{S}}$ was explained 
a long time ago in terms of "energy-limited" and "carbon-limited" substrates [40], no such explanation has ever been developed for anaerobic growth. The definite explanation has thus to await a deeper analysis based on the Second Law in the next section of this text.

\subsubsection{On-line monitoring and control of bioprocesses by heat dissipation measurements}

As explained earlier, the measurement of heat generation rates becomes easier at large scale due to the diminished surface-to-volume ratio. Such measurements therefore provide a simple way to monitor the biological activity of a culture and thus to control the bioprocess. This approach has been demonstrated on a 3001 pilot-scale bioreactor producing biological pesticides in India by Voisard et al. [8]. Later Türker [7] applied the method to a $100 \mathrm{~m}^{3}$ industrial bioreactor.

The best way to aid bioprocess monitoring by heat dissipation measurements is undoubtedly to combine them with other on-line measurements. In large-scale bioreactors, oxygen uptake and carbon dioxide evolution rates are often routinely monitored anyway. The same is true for the continuous determination of the consumption of acid and base to keep the $\mathrm{pH}$ constant. If on-line heat monitoring is added as well, the resulting four on-line rate measurements may be combined with typically five constraints resulting from balances for carbon, degree of reduction, nitrogen, charge, and energy to yield a considerably over-determined set of rates, at least for typical microbial bioprocess stoichiometry. It is therefore possible to check for gross measurement errors, to reconcile the data for more probable values, and to determine the unmeasured rates, including the growth rate, on line $[41,42]$.

\subsection{The Second Law: Free energy dissipation, driving force, and growth}

\subsubsection{Entropy and growth}

Growth of unicellular organisms such as microbes occurs spontaneously and is obviously a highly irreversible phenomenon. It must therefore be coupled with the production of entropy. In relation to growth reactions this seems contradictory, because growth reactions produce matter in a highly organized form from a set of very simple small molecules. One intuitively gets the impression that growth decreases the entropy rather than producing it. 
This contradiction may be resolved by contemplating an open-system entropy balance for the growing cell (Figure 8). The formulation of balances for the cell depicted in Figure 8 may be simplified with respect to the energy balance treated in Section 2.1 by assuming (i) that the surface of the cell is the only exchange port through which material may enter or leave, and that the cell operates at (ii) constant pressure and (iii) constant temperature. Simplification (i) is possible because the system does not include the whole culture vessel, but only the biomass contained in the broth. Allowing for these constraints, an entropy balance reads $[2,43]$

$$
\frac{\mathrm{d} S}{\mathrm{~d} t}=\frac{\dot{Q}}{T}+\sum_{i} \bar{s}_{i} \cdot \dot{n}_{i}-\bar{s}_{x} \cdot \dot{n}_{x}+\dot{S}_{\text {prod }} .
$$

According to this balance, the variation of entropy in the cell with time is given by the sum of all entropy fluxes exchanged with the environment plus the rate at which entropy is produced by irreversible processes $\left(\dot{S}_{\text {prod }}\right)$. Entropy may be exchanged with the environment due to heat transfer to or from the cell denoted by $\dot{Q} / T$ (d $Q_{\text {rev }} / T$ represents the entropy increase in closed systems). In open systems, entropy is also imported or exported through metabolites entering or leaving the cell. $\bar{s}_{i}$ denotes the partial molar entropy carried by the $i$-th metabolite and $\dot{n}_{i}$ its molar rate of exchange, whereby positive values indicate assimilation rates. Newly formed biomass is treated as a product of the cell leaving it at a C-molar rate of $\dot{n}_{x}$. Its partial molar entropy $\bar{s}_{x}$ tends to be low due to the high degree of organization of matter. The Second Law of Thermodynamics constrains the rate of entropy production by irreversible processes $\dot{S}_{\text {prod }}$ to non-negative values (Eq. (9)). It represents the actual driving force for the process:

$$
\dot{S}_{\text {prod }} \geq 0 \text {. }
$$

Due to constant entropy production at rate $\dot{S}_{\text {prod }}$ and due to the fact that newly formed cells of low entropy content leave the cell but have been synthesized by importing high-entropy metabolites, entropy could in principle accumulate in the cell and lead to thermal cell death or to structural disorganization. In order to avoid this, the cell must constantly export the excess entropy, i.e., it must keep $\mathrm{d} S / \mathrm{d} t$ at zero by making the sum of the first three terms on the right-hand side of Eq. (8) negative. This is precisely the role of catabolism. There are two ways in which catabolism can export excess entropy: First, by creating a large flux of small and/or gaseous waste molecules from the substrate, thereby exporting it in the form of chemical entropy and making $\sum_{i} \bar{s}_{i} \dot{n}_{i}$ strongly negative; and second, by releasing considerable amounts of heat, thereby making $\dot{Q} / T$ strongly negative. 


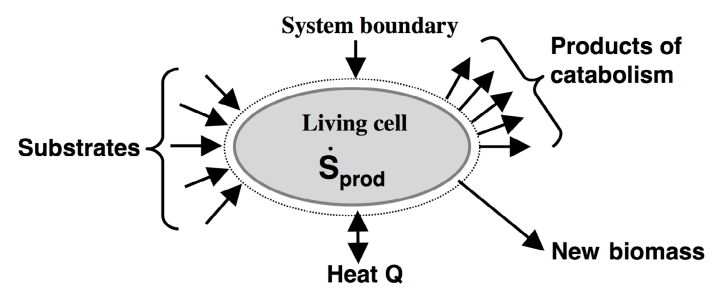

Figure 8. Illustration of an entropy balance for a growing cell. Reprinted with permission from von Stockar et al. [2].

This is the fundamental reason why virtually all living organisms constantly generate heat.

\subsubsection{Gibbs energy and growth}

If Eq. (8) is multiplied by $T$ and substracted from an enthalpy balance around the cell formulated in an analogous way to Eq. (8), a Gibbs energy balance results [2] (Figure 9). Assuming again constant pressure and temperature and accounting for only the cell surface as matter exchange site, it reads:

$$
\frac{\mathrm{d} G}{\mathrm{~d} t}=\dot{W}+\sum_{i} \mu_{i} \cdot \dot{n}_{i}-\mu_{x} \dot{n}_{x}-T \dot{S}_{\text {prod }}
$$

In Eq. (10), $\dot{W}$ stands for the power or work done on the cells, and $\mu_{i}$ and $\mu_{x}$ for the chemical potential of the $\mathrm{i}$-th metabolite and the newly grown cells $(\mathrm{kJ} / \mathrm{C}-\mathrm{mol})$ in the broth, respectively. The latter $\left(\mu_{\mathrm{X}}\right)$ tends to be high due to the low entropy of biomass. In order to avoid death, the cell has to keep $\mathrm{d} G / \mathrm{d} t$ at zero despite a constant loss of Gibbs energy through the newly formed biomass $\left(-\mu_{x} \dot{n}_{x}\right)$ and through dissipation or destruction of Gibbs energy represented by the term $\left(-T \dot{S}_{\text {prod }}\right)$, which can only be negative. In phototrophs, this loss is replenished by a positive $\dot{W}$ term in the form of photons. Chemotrophs on the other hand have a catabolism that feeds on high Gibbs energy substrates and release low energy waste products, thereby making $\sum_{i} \mu_{i} \dot{n}_{i}$ so positive that it overcompensates $-\mu_{x} \dot{n}_{x}$ and $-T \dot{S}_{\text {prod. }}$. The result is a continuous decrease of Gibbs energy in the surrounding medium.

The rate of Gibbs energy destruction in the surrounding medium may be found by using a molar balance for substituting the import/export rate of Gibbs energy in the balance above. Assuming again that the cell sur- 


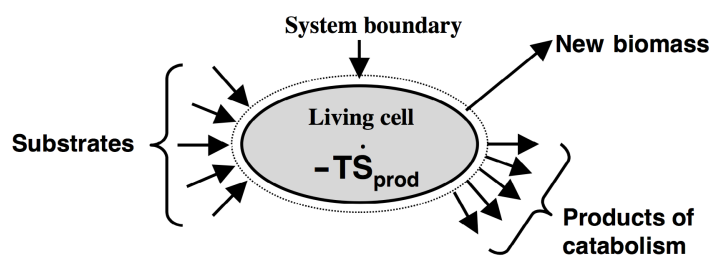

Figure 9. Illustration of a Gibbs energy balance for a growing cell. Reprinted with permission from von Stockar et al. [2].

face acts as the only exchange port, the molar balance (see Eq. (A.3), Appendix A) simplifies to

$$
\frac{\mathrm{d} n_{i}}{\mathrm{~d} t}=\dot{n}_{i}+v_{i} \cdot r_{\mathrm{X}} V
$$

where $\mathrm{d} n_{i} / \mathrm{d} t$ is zero because the cell works at steady state. The stoichiometric coefficients $v_{i}$ for a typical microbial growth reaction are given by Eq. (2). Solving this for $\dot{n}_{i}$ and substituting into Eq. (10) for a cell working at steady state, $(\mathrm{d} G / \mathrm{d} t=0)$ yields [43]

$$
\Delta_{r} G_{\mathrm{X}} \cdot r_{\mathrm{X}} V=\dot{W}-T \cdot \dot{S}_{\mathrm{prod}}
$$

with

$$
\Delta_{r} G_{\mathrm{X}} \equiv \sum_{i} v_{i} \mu_{i}=\Delta_{r} H_{\mathrm{X}}-T \Delta_{r} S_{\mathrm{X}}
$$

where $\Delta_{r} G_{\mathrm{X}}$ is the molar Gibbs reaction energy of the macrochemical reaction (2). The sum in Eq. (13) has to be performed over all constituents of the macrochemical Eq. (2) including biomass.

In non-photosynthetic growth $\dot{W}$ is usually zero. In this case, Eq. (12) shows that $\Delta_{r} G_{\mathrm{X}} \cdot r_{\mathrm{X}} V(\mathrm{~kJ} / \mathrm{s})$ reflects the rate at which cells export the entropy produced within them by irreversible processes into the fermentation medium. $\Delta_{r} G_{\mathrm{X}}$ must clearly be negative for growth to occur since $\dot{S}_{\text {prod }}$ can only be positive. $\Delta_{r} G_{\mathrm{X}} \cdot r_{\mathrm{X}} V$ therefore also reflects the rate at which Gibbs energy is dissipated by irreversible processes occurring in the cells. The Gibbs energy in the growth medium will thus decrease at a corresponding rate, as can be shown easily by a Gibbs energy balance over the whole bioreactor.

Due to its direct relationship with the rate of entropy generation in the case of $\dot{W}=0$ (Eq. (13)), $\Delta_{r} G_{\mathrm{X}}$ is called the driving force for its conjugate 
flux $r_{\mathrm{X}} V$, i.e., for growth. According to irreversible thermodynamics, it exerts a determining influence on the magnitude of this conjugate flux, i.e., on the growth rate. Equation (12) shows (for $\dot{W}=0$ ) that the more negative $\Delta_{r} G_{\mathrm{X}}$ is, the more entropy may be produced per biomass grown, which results in a higher growth rate according to irreversible thermodynamics. As shown by Eq. (13), the driving force has an enthalpic and an entropic part, which correspond to the export of entropy in the form of heat and in the form of high entropy molecules, respectively.

\subsubsection{The relationship between driving force and biomass yield}

The relationship between the driving force for microbial growth $\Delta_{r} G_{\mathrm{X}}$ and the biomass yield $Y_{\mathrm{X} / \mathrm{S}}$ is best understood by splitting the macrochemical reaction into a catabolic and an anabolic part (Figure 10). If the anabolic reactions in the cell, which are the ones actually synthesizing the new biomass from chemical compounds serving as carbon, nitrogen, and other sources, were considered independently of other processes, they would certainly not entail a strong decrease, but might rather give rise to an increase of Gibbs energy. Since biomass would then be in a state of higher Gibbs energy than the growth substrates, biomass could not be synthesized, but would rather have the tendency to decay into simpler molecules. In order to prevent this and to drive biomass synthesis "up-hill" against a potentially positive Gibbs energy gradient, anabolic reactions are coupled in live cells by biochemical mechanisms to a so-called energy yielding or catabolic reaction that is sufficiently exergonic to make the combination of the two reactions possible (Figure 10).

Whether the resulting overall change of Gibbs energy for this combination is really negative and by which amount is mainly determined by the biomass yield $Y_{\mathrm{X} / \mathrm{S}}$ (C-mole dry biomass synthesized per C-mole of carbon and energy substrate consumed). If the partial driving forces for the catabolic and biosynthetic reactions, $\Delta G_{\text {cat }}$ and $\Delta G_{\text {bios }}$ respectively, were known, the remaining driving force per mole of energy substrate consumed $\Delta_{r} G_{\mathrm{S}}$ could be calculated as a function of the biomass yield as follows [3]:

$$
\Delta_{r} G_{\mathrm{S}}=\left(1-Y_{\mathrm{X} / \mathrm{S}}\right) \cdot \Delta G_{\mathrm{cat}}+Y_{\mathrm{X} / \mathrm{S}} \cdot \Delta G_{\mathrm{bios}} \cdot
$$

In inefficient growth systems with small biomass yields, the "payload" would be small, and only a small amount of biomass would be produced per carbon and energy substrate consumed and $Y_{\mathrm{X} / \mathrm{S}}$ in Eq. (14) would remain small. However, the overall $\Delta_{r} G_{\mathrm{S}}$ would remain highly negative, be- 


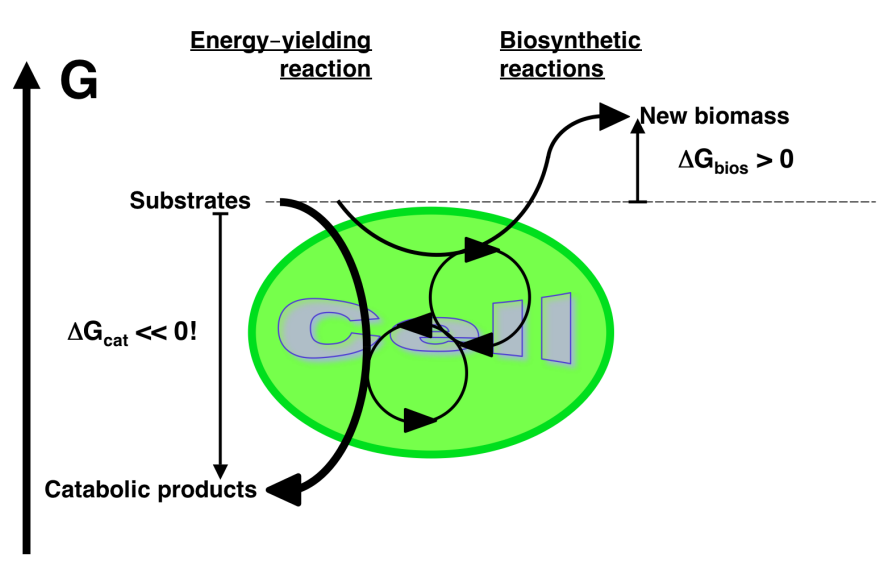

Figure 10. Gibbs energy transduction from energy-yielding to biosynthetic reactions. The latter may be bioenergetically "up-hill" and might proceed from biomass to substrate if left alone. In living cells, they are however coupled to an energy-yielding reaction and thus pulled up against their own driving force. The energy-yielding reaction has a strong driving force downwards. The degree of coupling in the sense of stoichiometry existing between the two determines the biomass yield, but also the amount of residual driving force, $\Delta G$, remaining for both reactions together.

cause the positive $\Delta G$ of anabolism would hardly make its weight felt on the downward pull of catabolism. In Eq. (14), the second right-hand term would only add a very small positive amount of Gibbs energy to the very highly negative first right-hand-side term. Growth would thus be vigorous but would only yield a small amount of biomass.

Conversely, in efficient growth with a high biomass yield, the load would be much more important and would neutralize a large part of the negative $\Delta G$ of catabolism, thereby leaving only a small negative $\Delta G$ for the overall process. On the other hand, a lot of biomass would be formed per substrate consumed and $Y_{\mathrm{X} / \mathrm{S}}$ would be high. At the limit, the "payload" could be so large that the two terms on the right-hand side of Eq. (14) would compensate each other. $\Delta_{r} G$ would then be zero and the two forces in equilibrium. This situation would define the highest $Y_{\mathrm{X} / \mathrm{S}}$ allowed by the Second Law of Thermodynamics. It would represent growth at the thermodynamic equilibrium, which would thus proceed at an infinitely slow rate.

It is obvious that real microbial growth systems must strike a compromise between two unfavorable extremes: A very low "payload" would provide a high overall driving force and the metabolism would proceed vigorously, but only little biomass would be formed and $Y_{\mathrm{X} / \mathrm{S}}$ would be 
small. If, on the other hand, growth occurred too close to thermodynamic equilibrium, the biomass yield would indeed be optimal, but growth would proceed so slowly that the organism would always be outgrown by competitors. Real organisms must therefore have a $\Delta_{r} G_{\mathrm{X}}$ and a $Y_{\mathrm{X} / \mathrm{S}}$ somewhere between these two extremes. This will later be illustrated on the basis of real data (cf. Figure 12).

\section{Quantitative investigation of the relationship between heat generation, Gibbs energy dissipation, and biomass yield}

\subsection{Calculation procedures for predicting the energy dissipation as a function of biomass yield}

\subsubsection{General equations}

Over many years, energy dissipation and biomass yields have been measured for a variety of microbial growth systems in the laboratory of the author $[2,3,6,43]$. In these studies, the dissipation of energy in the form of heat was directly measured in a reaction calorimeter and compared with predictions from an energy balance. The Gibbs energy dissipation was evaluated based on the observed stoichiometry.

For these investigations, the enthalpy and the Gibbs energy of reaction had to be evaluated based on their definition:

$$
\begin{aligned}
\Delta_{r} G^{0} & =-\sum v_{i} \Delta_{\mathrm{c}} G_{i}^{0}, \\
\Delta_{r} H^{0} & =-\sum v_{i} \Delta_{\mathrm{c}} H_{i}^{0} .
\end{aligned}
$$

The stoichiometric coefficients $v_{i}$ are defined by the yield factors in Eq. (2) and must be determined experimentally. The enthalpies and Gibbs energies of combustion ( $\Delta_{\mathrm{c}} H_{i}^{0}$ and $\Delta_{\mathrm{c}} G_{i}^{0}$, respectively) were known from thermodynamic tables. A simplification has however been introduced in formulating Eq. (15). It consists in replacing all Gibbs energies and enthalpies of reaction by their standard values. This is justified because in processes involving whole cells the concentration dependency is usually negligible as compared with the standard value.

The heat of combustion for dried biomass was determined by direct measurements in a combustion calorimeter [44]. However, the Gibbs energy of combustion of dry biomass cannot be measured experimentally. There 
are many more or less speculative estimations for $\Delta_{\mathrm{c}} G_{\mathrm{X}}^{0}$ in the literature, which are mostly based on model estimates for the entropy of dry biomass (for a review, see von Stockar and Liu [43]). The only value of the absolute entropy in dry biomass with an experimental basis was determined by lowtemperature calorimetry by Battley et al. [46]. Combining this with heat of combustion data yields an estimation for the Gibbs energy of combustion of dry biomass of $\Delta_{\mathrm{c}} G_{\mathrm{X}}^{0}=-515 \mathrm{~kJ} / \mathrm{C}$-mol for $S$. cerevisiae. Since the elemental composition of dry biomass is known to be fairly constant from one strain to another, this value may also be used for other types of biomass as a good estimate of $\Delta_{\mathrm{c}} G_{x}^{0}$.

In order to use Eq. (15), one needs numerical values for the stoichiometric coefficients or yields in Eq. (2). The biomass yields $Y_{\mathrm{X} / \mathrm{S}}$ were determined experimentally, and the product yield $Y_{\mathrm{P} / \mathrm{X}}$ from a degree of reduction balance. $Y_{\mathrm{N} / \mathrm{X}}$ turns out to be equal to $x_{\mathrm{N}}$ from a nitrogen balance, and the other yields are often immaterial if the completely combusted state is used as a reference state.

\subsubsection{Simplified calculations based on a formal energy-yielding and a biosynthetic reaction}

The influence of the biomass yield on both the driving force $\Delta_{r} G_{\mathrm{X}}$ and its enthalpic component $\Delta_{r} H_{\mathrm{X}}$ becomes clearer if the stoichiometry of the overall growth process (Eq. (2)) is split up formally in an energy-yielding and a biosynthetic reaction as shown in Figure 10.

The underlying assumption of Figure 10 is that all carbon compounds in biomass are directly synthesized from the carbon substrate. Compared to real biochemistry, this is obviously a gross oversimplification. In reality, hundreds of different constituent components of biomass are derived from different points along the catabolic series of reactions. Hence, trying to use a real formulation of anabolism is unpractical due to the complexity of its details, which are often unknown [47]. Rather, a formal though somewhat unrealistic split such as the one in Figure 10 may be assumed. Although $\Delta G_{\text {bios }}$ may not bear a realistic signification, prediction will be correct provided that $\Delta G$ for biosynthesis and catabolism are not used separately.

Therefore, many formal ways of splitting the overall growth stoichiometry into catabolism and a formal anabolic reaction may be proposed and have been used in the literature, including the one shown in Figure 10. A numerical example of an experimentally observed growth stoichiometry and different ways to split it into catabolism and biosynthesis is given 


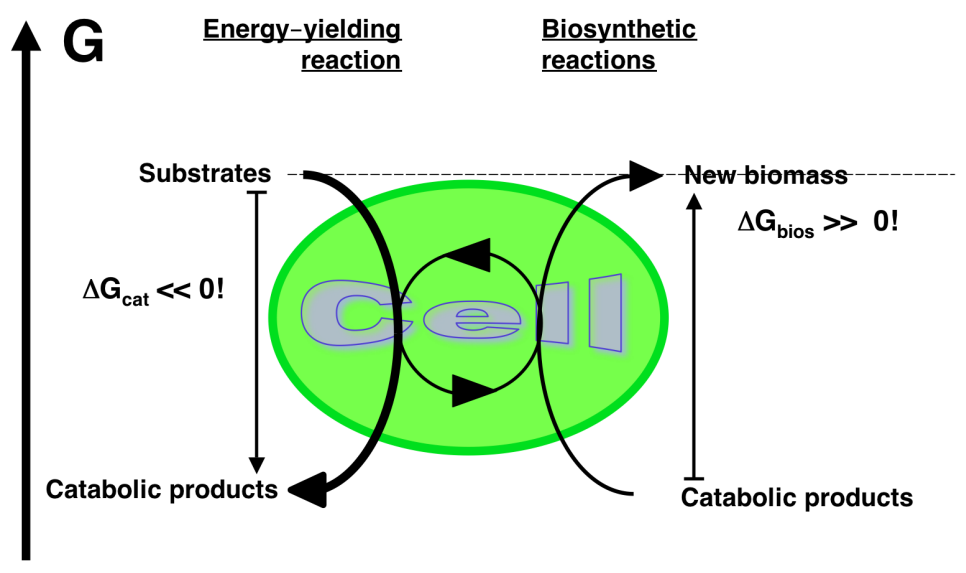

Figure 11. Gibbs energy transduction from energy-yielding to biosynthetic reactions. In formulating the biosynthetic reaction it was assumed that all of the substrate is catabolized first and biomass then synthesized from catabolic products. Reprinted with permission from von Stockar et al. [2].

in Appendix B. One way treated in Appendix B to perform this split is to assume that all of the carbon and energy substrate is first catabolized and some of the products of catabolism are then used to synthesize biomass, as depicted in Figure 11.

This split is obviously even less realistic, but it affords particularly easy computational procedures. This is why the split depicted in Figure 11 will be used for all further calculation in this contribution. It has been shown by von Stockar et al. [3] that although different formal biosynthetic reactions such as proposed in Figures 10 and 11 assign different values to $\Delta G_{\text {bios }}$, the results obtained for $\Delta_{r} G_{\mathrm{X}}$ (or $\Delta_{r} H_{\mathrm{X}}$ ) as a function of the biomass yield and $\Delta G_{\text {bios }}$ will always be the same, provided Eq. (14) and its enthalpy analog are correctly adapted to the chosen formal biosynthetic reaction. It should also be kept in mind that the same results could always be obtained by calculating the stoichiometric coefficients in Eq. (2) from an experimentally determined biomass yield by applying elemental balances, and then computing $\Delta_{r} G_{\mathrm{X}}$ (or $\Delta_{r} H_{\mathrm{X}}$ ) based on Eq. (15). The advantages of the formalism proposed in this section lie in the graphical visualizations it permits, such as Figures 10 and 11, as well as in the easiness with which the necessary equations are written and manipulated.

Following the split proposed in Figure 11, the catabolic and anabolic reactions have to be formulated as shown in the following equations (for an 
example, see Appendix B):

Reaction (a) (Catabolism):

$$
\mathrm{S}+Y_{\mathrm{A}}^{\mathrm{a}} \mathrm{A} \Rightarrow Y_{\mathrm{P}}^{\mathrm{a}} \mathrm{P}+Y_{\mathrm{SOX}}^{\mathrm{a}} \mathrm{SOX} \quad \Delta H_{\mathrm{a}}^{0}, \Delta G_{\mathrm{a}}^{0},
$$

Reaction (b) (Biosynthesis):

$$
Y_{\mathrm{P}}^{\mathrm{b}} \mathrm{P}+Y_{\mathrm{SOX}}^{\mathrm{b}} \mathrm{SOX}+Y_{\mathrm{N}}^{\mathrm{b}} \mathrm{NH}_{3} \Rightarrow \mathrm{X}+Y_{\mathrm{A}}^{\mathrm{b}} \mathrm{A} \quad \Delta H_{\mathrm{b}}^{0}, \Delta G_{\mathrm{b}}^{0} .
$$

These equations have been generalized in order to enable them to cover also cases in which the carbon and the energy substrate are not identical. In Eq. (16), S stands for the energy source in the growth reaction, A for the electron acceptor, $\mathrm{P}$ for the reduced form of $\mathrm{A}$, and SOX denotes the oxidized form of $\mathrm{S}$. In heterotrophic growth $\mathrm{S}$ is also the carbon source, yielding $\mathrm{H}_{2} \mathrm{O}$ as $\mathrm{P}$ and $\mathrm{CO}_{2}$ as $\mathrm{SOX}$, whereas in autotrophic growth $\mathrm{A}$, which then is $\mathrm{CO}_{2}$, provides the carbon. In fermentative or reductive growth, $\mathrm{A}$ and $\mathrm{S}$ are the same compound (cf. Table 3).

In a vast majority of cases, only $\mathrm{S}, \mathrm{X}, \mathrm{P}$, and $\mathrm{NH}_{3}$ have a heat of combustion and a Gibbs energy of combustion different from zero, and thus only those compounds are relevant for calculating the enthalpy and the Gibbs energy of reaction. The values for $Y_{\mathrm{P}}^{\mathrm{a}}$ and for $Y_{\mathrm{P}}^{\mathrm{b}}$ needed for this calculation are obtained in a straightforward manner by applying a degree of reduction balance; the one for $Y_{\mathrm{N}}^{\mathrm{b}}$, by applying a nitrogen balance.

Table 3. Examples of energy source, electron acceptor, reduced electron acceptor, and oxidized energy source for the growth systems discussed in

\begin{tabular}{|c|c|c|c|c|}
\hline Examples of: & $\begin{array}{l}\text { Energy } \\
\text { source }\end{array}$ & $\begin{array}{l}\text { Electron } \\
\text { acceptor }\end{array}$ & $\begin{array}{l}\text { Reduced } \\
\text { electron } \\
\text { acceptor }\end{array}$ & $\begin{array}{l}\text { Oxidized } \\
\text { energy } \\
\text { source }\end{array}$ \\
\hline Metabolism & $s$ & A & $\mathbf{P}$ & sox \\
\hline Aerobic growth & glucose & $\mathrm{O}_{2}$ & $\mathrm{H}_{2} \mathrm{O}$ & $\mathrm{CO}_{2}$ \\
\hline Ethanol fermentation & glucose & glucose & EtOH & $\mathrm{CO}_{2}$ \\
\hline Lactic acid fermentation & lactose & lactose & lactate & lactate \\
\hline $\begin{array}{l}\text { Acetotrophic } \\
\text { Methanogenesis }\end{array}$ & $\mathrm{CH}_{3} \mathrm{COOH}$ & $\mathrm{CH}_{3} \mathrm{COOH}$ & $\mathrm{CH}_{4}$ & $\mathrm{CO}_{2}$ \\
\hline $\begin{array}{l}\text { Autotrophic } \\
\text { methanogenesis }\end{array}$ & $\mathrm{H}_{2}$ & $\mathrm{CO}_{2}$ & $\mathrm{CH}_{4}$ & $\mathrm{H}_{2} \mathrm{O}$ \\
\hline
\end{tabular}
this review. Adapted from von Stockar and Liu [43]. 
As a result, the enthalpies and Gibbs energies of reaction (16a) (catabolism) and reaction (16b) (anabolism) may be computed very simply as:

$$
\begin{aligned}
& \Delta G_{\mathrm{a}}^{0}=\Delta_{\mathrm{c}} G_{\mathrm{S}}^{0}-\frac{\gamma_{\mathrm{S}}}{\gamma_{\mathrm{P}}} \Delta_{\mathrm{c}} G_{\mathrm{P}}^{0}, \\
& \Delta G_{\mathrm{b}}^{0}=\frac{\gamma_{\mathrm{X}}}{\gamma_{\mathrm{P}}} \Delta_{\mathrm{c}} G_{\mathrm{P}}^{0}-\Delta_{\mathrm{c}} G_{\mathrm{X}}^{0}+x_{\mathrm{N}} \cdot \Delta_{\mathrm{c}} G_{\mathrm{N}}^{0}, \\
& \Delta H_{\mathrm{a}}^{0}=\Delta_{\mathrm{c}} H_{\mathrm{S}}^{0}-\frac{\gamma_{\mathrm{S}}}{\gamma_{\mathrm{P}}} \Delta_{\mathrm{c}} H_{\mathrm{P}}^{0}, \\
& \Delta H_{\mathrm{b}}^{0}=\frac{\gamma_{\mathrm{X}}}{\gamma_{\mathrm{P}}} \Delta_{\mathrm{c}} H_{\mathrm{P}}^{0}-\Delta_{\mathrm{c}} H_{\mathrm{X}}^{0}+x_{\mathrm{N}} \cdot \Delta_{\mathrm{c}} H_{\mathrm{N}}^{0} .
\end{aligned}
$$

The stoichiometry for the growth reaction as a whole is obtained by dividing Eq. (16a) by $Y_{\mathrm{X} / \mathrm{S}}$ and adding the result to Eq. (16b):

$$
\frac{1}{Y_{\mathrm{X} / \mathrm{S}}} \mathrm{S}+Y_{\mathrm{A} / \mathrm{X}} \mathrm{A}+Y_{\mathrm{N} / \mathrm{X}} \mathrm{NH}_{3} \rightarrow X+Y_{\mathrm{P} / \mathrm{X}} \mathrm{P}+Y_{\mathrm{SOX} / \mathrm{X}} \mathrm{SOX} .
$$

Performing the same operation on $\Delta G_{\mathrm{a}}^{0}$ and $\Delta G_{\mathrm{b}}^{0}$ yields the relationship between the driving force of the entire growth process and the biomass yield:

$$
\Delta_{r} G_{\mathrm{X}}^{0}=\frac{1}{Y_{\mathrm{X} / S}} \Delta G_{\mathrm{a}}^{0}+\Delta G_{\mathrm{b}}^{0} .
$$

A similar relationship may be derived for the standard heat of reaction and the biomass yield:

$$
\Delta_{r} H_{\mathrm{X}}^{0}=\frac{1}{Y_{\mathrm{X} / \mathrm{S}}} \Delta H_{\mathrm{a}}^{0}+\Delta H_{\mathrm{b}}^{0} .
$$

$\Delta_{r} G_{\mathrm{X}}^{0}$ and $\Delta_{r} H_{\mathrm{X}}^{0}$ are the overall Gibbs energy and enthalpy change, respectively, expressed in $\mathrm{kJ}$ per $\mathrm{C}$-mole biomass grown.

\subsection{Aerobic growth}

In several experimental research projects $[3,6,43]$ the biomass yields were measured for aerobic batch and chemostat cultures under very many different growth conditions at varying specific growth rates, and $\Delta_{r} G_{\mathrm{X}}^{0}$ values were calculated according to Eq. (20a). In addition, the enthalpic part of the driving force of growth, $\Delta_{r} H_{\mathrm{X}}^{0}$, was measured directly by applying reaction calorimetry and compared with values computed according to 
Eq. (20b). The $\Delta_{r} G_{\mathrm{X}}^{0}$ and $\Delta_{r} H_{\mathrm{X}}^{0}$ values are plotted against the corresponding biomass yields in Figure 12, along with Eqs. (20a) and (20b) (thick and thin curve, respectively). The thick curve shows clearly that the driving force $-\Delta_{r} G_{\mathrm{X}}^{0}$ for growth decreases as a function of assumed biomass yields, as predicted qualitatively in Section 2.2.3.

The corresponding thin line for $\Delta_{r} H_{\mathrm{X}}^{0}$ is very close to the one for Gibbs energy, which indicates that $T \Delta_{r} S_{\mathrm{X}}^{0}$, which ought to separate the two lines, is close to zero. It may be concluded that aerobic growth on glucose is driven almost entirely by an enthalpy change, or that the entropy produced by irreversible processes is exported practically exclusively in the form of heat rather than in the form of small waste molecules (cf. end of Section 2.2.1). The maximal value of $Y_{\mathrm{X} / \mathrm{S}}$ allowed by the Second Law, i.e., the yield that reduces $-\Delta_{r} G_{\mathrm{X}}^{0}$ to zero, is calculated to be close to $1 \mathrm{C}$ $\mathrm{mol} / \mathrm{C}$-mol. Since the thin line corresponding to $\Delta_{r} H_{\mathrm{X}}^{0}$ is above the line for $\Delta_{r} \mathrm{G}_{X}^{0}$, this means that, from a purely thermodynamic point of view,

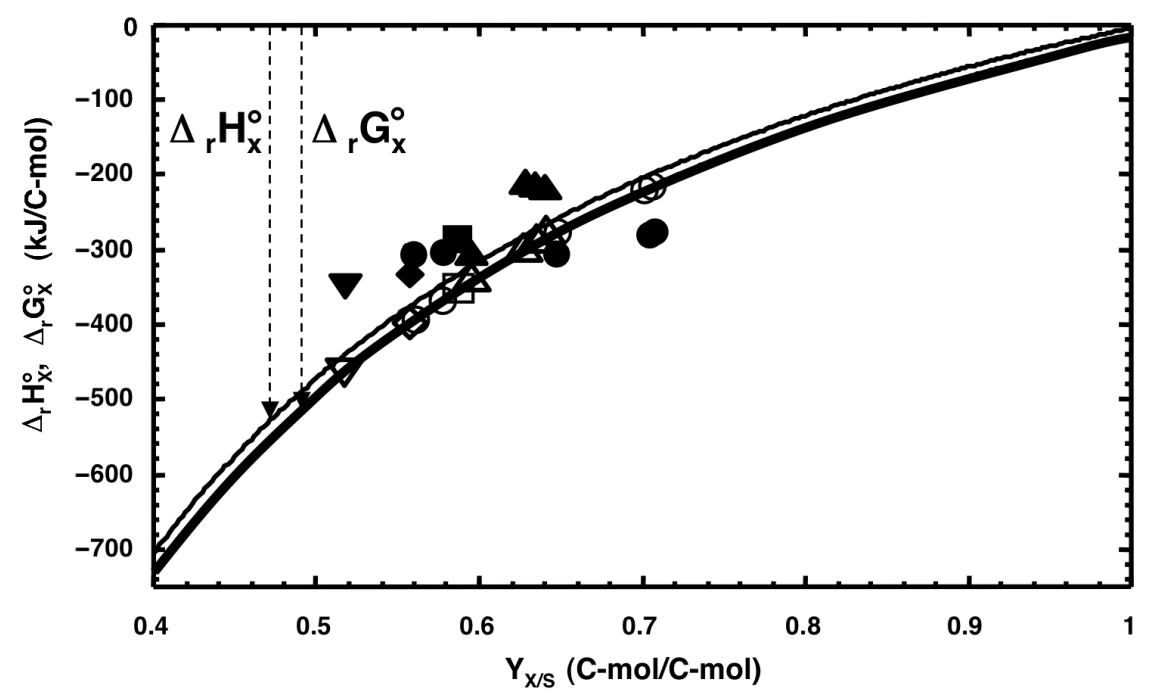

Figure 12. Standard Gibbs energies $\left(\Delta_{r} G_{\mathrm{X}}^{0}\right.$, calculated) and standard enthalpies $\left(\Delta_{r} H_{\mathrm{X}}^{0}\right.$, measured) of the growth reaction versus biomass yields for aerobic growth on glucose. Thick line, $\Delta_{r} G_{\mathrm{X}}^{0}$ calculated from Eq. (20a); thin line, $\Delta_{r} H_{\mathrm{X}}^{0}$ calculated from Eq. (20b); open symbols, $\Delta_{r} G_{\mathrm{X}}^{0}$ calculated for experimental biomass yields; full symbols, $\Delta_{r} H_{\mathrm{X}}^{0}$ measured by calorimetry; dots/circles, K. marxianus; squares, C. utililis; triangles, S. cerevisiae; diamonds, C. pseudotropicalis; inverted triangles, E. coli. Adapted from von Stockar et al. [2]. 
a very slightly endothermic aerobic growth process would be possible with a biomass yield close to unity and practically no release of carbon dioxide.

Despite the enormous differences in strains, growth conditions and rates, the actually measured biomass yields as well as the measured reaction enthalpies fall within quite a narrow range. The biomass yields are quite high, yet still far away from the theoretical maximum. This probably reflects the compromise between two unfavorable extremes discussed in Section 2.2.3. The driving forces $\Delta_{r} G_{\mathrm{X}}^{0}$ consequently do not vary widely and are comprised between -250 and $-500 \mathrm{~kJ}$ per $\mathrm{C}$-mole of dry biomass grown. The measured $\Delta_{r} H_{\mathrm{X}}^{0}$ values fall into a similar range. If the energy balances had been closed, the respective points should lie on the thin line, representing the predictions obtained from Eq. (20b). (The open symbols fall on the thick line, because $\Delta_{r} G_{\mathrm{X}}^{0}$ values cannot be measured but were calculated from measured $Y_{\mathrm{X} / \mathrm{S}}$ values in the same way as the thick line (Eq. (20a)).)

\subsection{Ethanol fermentation}

Figure 13 represents a similar plot as Figure 12, but for anaerobic growth of $K$. marxianus and $S$. cerevisiae. The catabolic reaction produces ethanol according to

$$
\mathrm{C}_{6} \mathrm{H}_{12} \mathrm{O}_{6} \rightarrow 2 \mathrm{CH}_{3} \mathrm{CH}_{2} \mathrm{OH}+2 \mathrm{CO}_{2} \text {. }
$$

The thin line representing $\Delta_{r} H_{\mathrm{X}}^{0}$ is now widely separated from the thick Gibbs energy of reaction line and indicates much lower negative values. The measured reaction enthalpies were around $-100 \mathrm{~kJ} / \mathrm{C}$-mol only and confirm the calculated line quite well. Despite this dramatically lower heat generation, vigorous growth occurred and the calculated Gibbs energy change still reached between -240 and $-340 \mathrm{~kJ}$ per C-mole of dry biomass grown. The separation of $\Delta_{r} G_{\mathrm{X}}^{0}$ and $\Delta_{r} H_{\mathrm{X}}^{0}$ shows that a large $T \Delta_{r} S_{\mathrm{X}}^{0}$ term contributed to the total driving force. In this case, about $2 / 3$ of the driving force was entropic and the same fraction of entropy generated in the cells was exported as chemical entropy in the form of the small molecules generated by the catabolic reaction (see Eq. (21)).

It is noteworthy that the Gibbs energy change of this culture is still of the same order as the ones observed for aerobic growth, despite the fact that the thick line is located completely differently in the plot. Due to the much smaller Gibbs energy change of the catabolic reaction, Eq. (16a), this line now indicates much less negative $\Delta_{r} G_{\mathrm{X}}^{0}$ for a given biomass yield. It could be hypothesized that the biomass yields in anaerobiosis are reduced to an extent permitting the culture to maintain the same overall driving force for growth. 


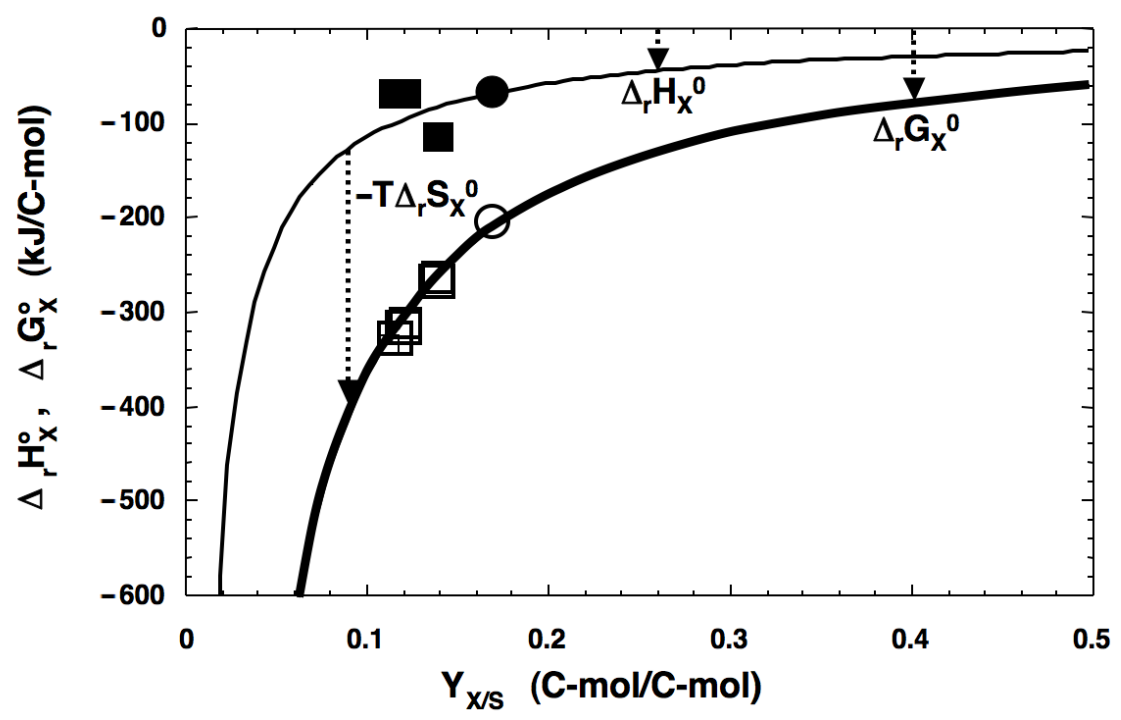

Figure 13. Standard Gibbs energies $\left(\Delta_{r} G_{\mathrm{X}}^{0}\right.$, calculated) and standard enthalpies $\left(\Delta_{r} H_{\mathrm{X}}^{0}\right.$, measured $)$ of the growth reaction versus biomass yields for ethanol fermentation on glucose. Thick line, $\Delta_{r} G_{\mathrm{X}}^{0}$ calculated from Eq. (20a); thin line, $\Delta_{r} H_{\mathrm{X}}^{0}$ calculated from Eq. (20b); open symbols, $\Delta_{r} G_{\mathrm{X}}^{0}$ calculated for experimental biomass yields; full symbols, $\Delta_{r} H_{\mathrm{X}}^{0}$ measured by calorimetry; dots/circles, S. cervisiae; squares, K. marxianus. Adapted from von Stockar and Liu [43].

\subsection{Lactic acid fermentation}

Figure 14 represents the driving forces as a function of biomass yields for anaerobic growth of Lactobacillus helveticae, which generates its energy from the following catabolic reaction [48]:

$$
\mathrm{C}_{6} \mathrm{H}_{12} \mathrm{O}_{6} \rightarrow 2 \mathrm{CH}_{3} \mathrm{CHOHCOOH} .
$$

Although this catabolic reaction might be expected to export the excess entropy almost solely by producing small molecules from large ones, Figure 14 shows a majority of the driving force to be enthalpic. The reason is the fact that at the growth-sustaining $\mathrm{pH}$, lactic acid is immediately neutralized, thereby yielding a considerable amount of heat. This neutralization reaction has been taken into account when calculating the $-\Delta_{r} G_{\mathrm{X}}^{0}$ curve in Figure 14, and it may be seen that the Gibbs energy driving forces resulting from the measured biomass yields are again of the same order of magnitude as in the previous cases. 


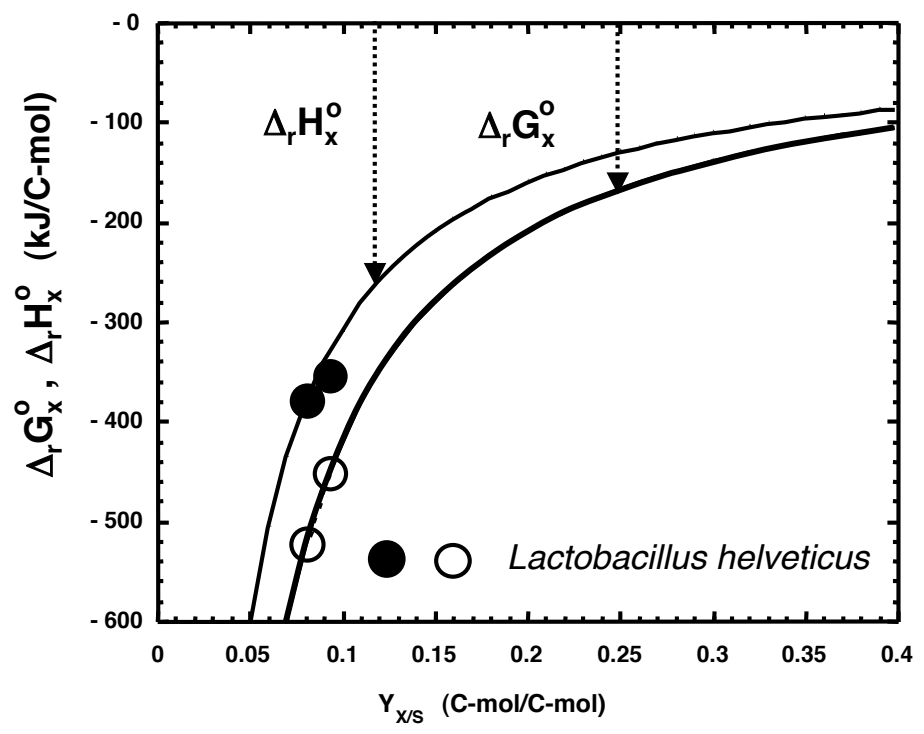

Figure 14. Standard Gibbs energies $\left(\Delta_{r} G_{X}^{0}\right.$, calculated) and standard enthalpies $\left(\Delta_{r} H_{\mathrm{X}}^{0}\right.$, measured) of the growth reaction versus biomass yields for homolactic fermentation on glucose by $L$. helveticus. Thick line, $\Delta_{r} G_{\mathrm{X}}^{0}$ calculated from Eq. (20a); thin line, $\Delta_{r} H_{\mathrm{X}}^{0}$ calculated from Eq. (20b); open symbols, $\Delta_{r} G_{\mathrm{X}}^{0}$ calculated for experimental biomass yields; full symbols, $\Delta_{r} H_{\mathrm{X}}^{0}$ measured by calorimetry. Adapted from von Stockar et al. [2].

\subsection{Acetotrophic methanogenesis}

The most extreme case of entropy-driven growth investigated as yet are anaerobic cultures of the bacterium Methanosarcina barkeri, which derives its energy from the following reaction (J. S. Liu et al. [49]):

$$
\mathrm{CH}_{3} \mathrm{COOH} \Rightarrow \mathrm{CH}_{4}+\mathrm{CO}_{2} \text {. }
$$

As may be seen in Figure 15, the $\Delta_{r} H^{0}$ curve is not only dramatically separated from the $\Delta_{r} G^{0}$ curve, but it is even above the zero line. This means that this culture not only does not produce any heat, but on the contrary absorbs heat and cools its environment down. As shown by the solid squares on the line, calorimetric measurements confirmed the endothermic nature of this culture and corresponded well with the calculated predictions. This is so far the only experimental demonstration of the existence of an endothermic life form. M. barkeri exports the excess entropy by turning one molecule in the aqueous state into two gaseous molecules, which increases the entropy in the bioreactor considerably. 


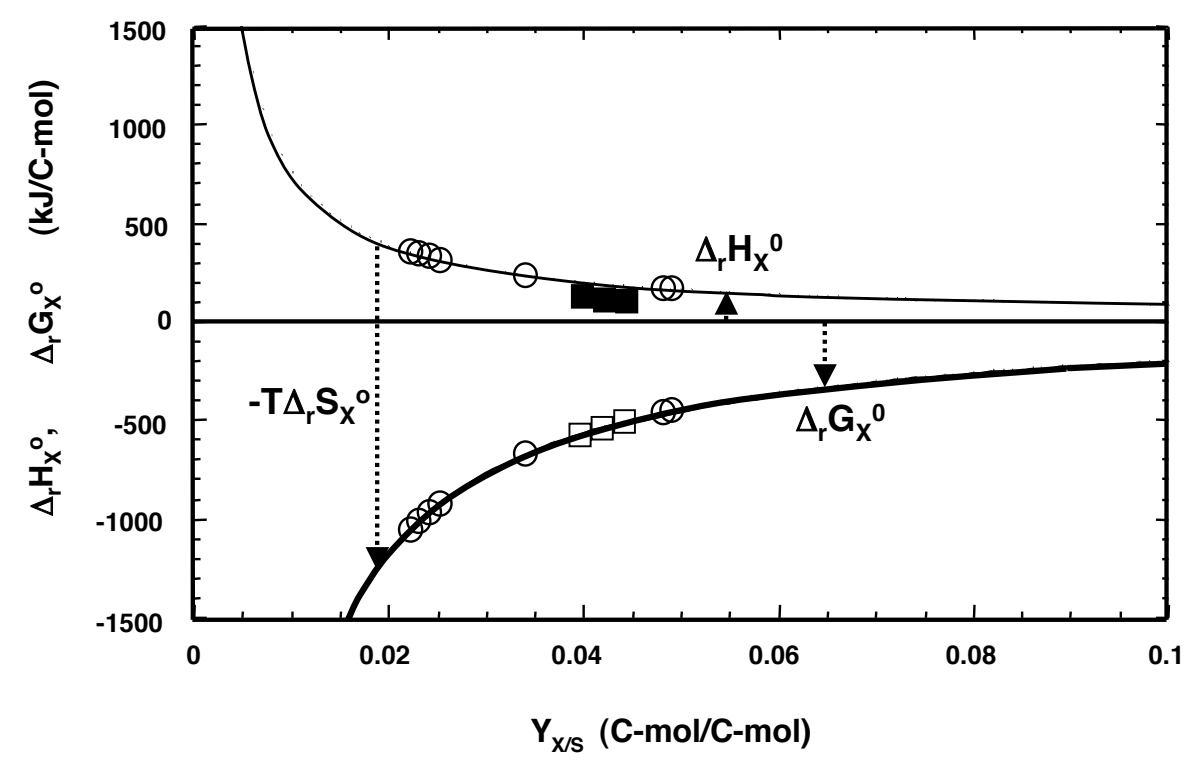

Figure 15. Standard Gibbs energies $\left(\Delta_{r} G_{\mathrm{X}}^{0}\right.$, calculated) and standard enthalpies $\left(\Delta_{r} H_{\mathrm{X}}^{0}\right.$, calculated or measured) of the growth reaction versus biomass yields for acetotrophic methanogenesis by M. barkeri. Thick line, $\Delta_{r} G_{\mathrm{X}}^{0}$ calculated from Eq. (20a); thin line, $\Delta_{r} H_{\mathrm{X}}^{0}$ calculated from Eq. (20b); open symbols, $\Delta_{r} G_{\mathrm{X}}^{0}$ and $\Delta_{r} H_{\mathrm{X}}^{0}$ calculated for experimental biomass yields; full symbols, $\Delta_{r} H_{\mathrm{X}}^{0}$ measured by calorimetry; squares, this work; circles, literature values. Adapted from Liu et al. [49].

The corresponding driving force for growth is sufficiently large to permit the culture to grow despite the fact that it must excrete waste products $\left(\mathrm{CH}_{4}\right.$ and $\mathrm{CO}_{2}$ ) of higher chemical energy than the feedstock (acetate), thereby forcing it to absorb the difference in the form of heat. Nevertheless, with $-500 \mathrm{~kJ} / \mathrm{C}$-mol the overall driving force $\Delta_{r} G_{\mathrm{X}}^{0}$ is again of the same order of magnitude as in all the other cases.

Entropy export in the form of producing gaseous molecules has been found potentially important in other growth processes, such as photosynthesis [50].

\subsection{Autotrophic methanogenesis}

Figure 16 shows the same calculation as Figure 15, but for the strictly anaerobic growth of Methanobacterium thermoautotrophicum on $\mathrm{H}_{2}$ as electron donor. The catabolic reaction for this culture is

$$
\mathrm{CO}_{2}+4 \mathrm{H}_{2} \Rightarrow \mathrm{CH}_{4}+2 \mathrm{H}_{2} \mathrm{O} \text {. }
$$




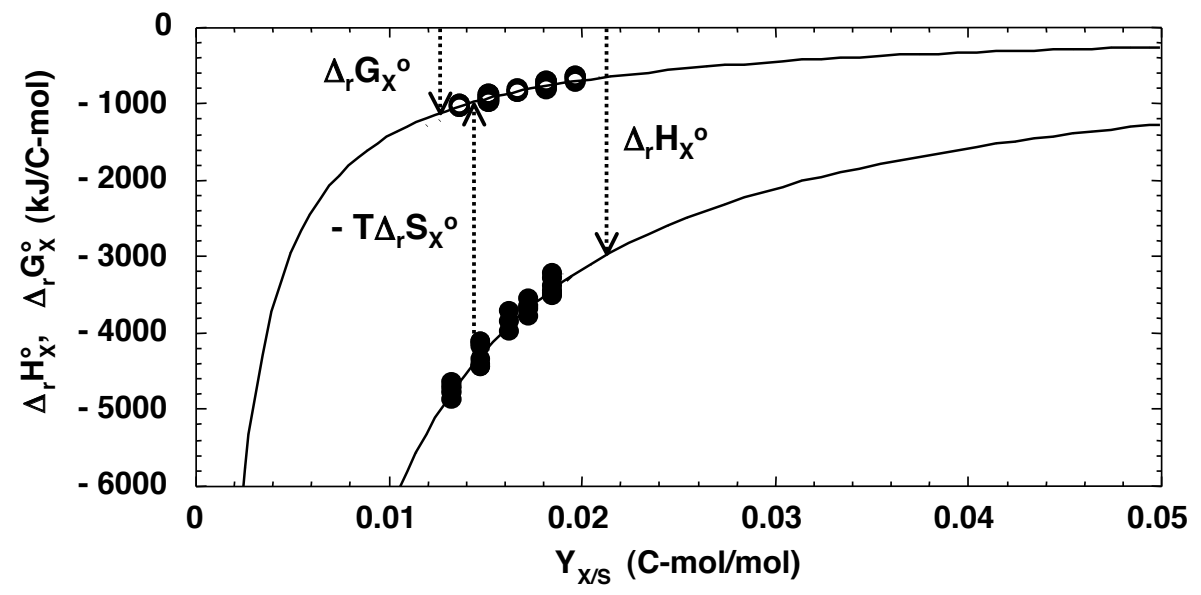

Figure 16. Standard Gibbs energies $\left(\Delta_{r} G_{\mathrm{X}}^{0}\right.$, calculated) and standard enthalpies $\left(\Delta_{r} H_{\mathrm{X}}^{0}\right.$, calculated or measured) of the growth reaction versus biomass yields for autotrophic methanogenesis by Methanobascterium thermoautotrophicum. Thick line, $\Delta_{r} G_{\mathrm{X}}^{0}$ calculated from Eq. (20a); thin line, $\Delta_{r} H_{\mathrm{X}}^{0}$ calculated from Eq. (20b); open symbols, $\Delta_{r} G_{\mathrm{X}}^{0}$ calculated for experimental biomass yields; full symbols, $\Delta_{r} H_{\mathrm{X}}^{0}$ measured by calorimetry. Adapted from von Stockar and Liu [43].

Here, again, a relatively large $T \Delta_{r} S_{\mathrm{X}}^{0}$ separates $\Delta_{r} H_{\mathrm{X}}^{0}$ from $\Delta_{r} G_{\mathrm{X}}^{0}$, but in this case, the entropy change is negative. The decrease of entropy, resulting from the conversion of many small gas molecules $\left(\mathrm{H}_{2}\right.$ !) into larger ones, must be compensated for by a very large degradation of chemical energy into heat $\left(\Delta_{r} H_{\mathrm{X}}^{0}\right)$. Therefore, the heat yield $Y_{Q / \mathrm{X}}(\mathrm{ca} 4000 \mathrm{~kJ} / \mathrm{C}-\mathrm{mol})$ is many times higher than for aerobic growth [51]. Methanogenesis from $\mathrm{H}_{2}$ and $\mathrm{CO}_{2}$ therefore appears to be characterized by an extremely high enthalpic driving force which serves to counteract the entropy decrease occurring when $\mathrm{H}_{2}$ and $\mathrm{CO}_{2}$ are transformed into methane. The Gibbs energy dissipation ranges from about -800 to $-1000 \mathrm{~kJ} / \mathrm{C}-\mathrm{mol}$ and is also much higher than for aerobic growth (about $-400 \mathrm{~kJ} / \mathrm{C}-\mathrm{mol}$ ).

\subsection{The relationship between heat generation and free energy dissipation}

According to the foregoing sections, microorganisms need to dissipate a certain amount of Gibbs energy corresponding to a certain driving force $\Delta_{r} G_{\mathrm{X}}^{0}$ in order to grow at a reasonable rate. In all cases discussed above 
with the exception of autotrophic methanogenesis, this dissipation fell into the range of about -250 to $-500 \mathrm{~kJ} / \mathrm{C}$-mol. The relationship between this dissipation and the heat generation of microbial cultures depends on how the driving force is subdivided into an enthalpic and an entropic part.

The driving force for growth $\Delta_{r} G_{\mathrm{X}}^{0}$ and its subdivision into an enthalpic and an entropic part are depicted schematically for different types of energy metabolism in Figure 17. The overall driving force itself, which has been found to often fall into a relatively narrow range, appears symbolically at the left of this figure. The bars and arrows further to the right demonstrate, however, that the separation of the driving force into an enthalpic and an entropic part differs widely from one case to another. Aerobic respiration, for instance, is enthalpy driven practically to $100 \%$ with the $T \Delta_{r} S_{\mathrm{X}}^{0}$ term (arrows) being insignificant. Fermentative metabolism, shown on the left of respiration, has lesser $-\Delta_{r} H_{\mathrm{X}}^{0}$ values because no external electron acceptors are involved. However, fermentations invariably rip substrate molecules apart into smaller entities, thereby generating an increase of chemical entropy in the medium that can be harnessed as driving force. In the case of $M$. barkeri, shown just right of the overall driving force $\Delta_{r} G_{\mathrm{X}}^{0}$, this entropic driving force has to overcome a positive enthalpy

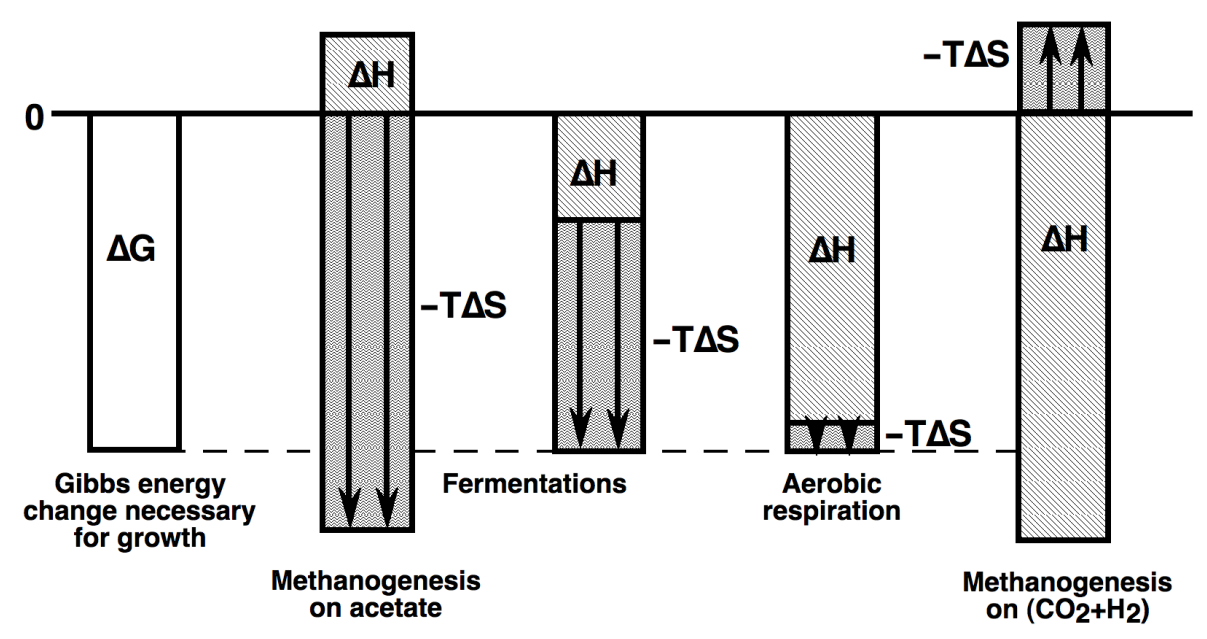

ENTROPY-DRIVEN GROWTH

Enthalpy-retarded

ENTHALPY-DRIVEN GROWTH

Entropy-retarded

Figure 17. Schematic representation of enthalpic and entropic contribution to the driving force of microbial growth according to $\Delta G^{0}=\Delta H^{0}-T \Delta S^{0}$. Adapted from Von Stockar and Liu [43]. 
change, which makes the culture endothermic and acts as a partial driving force in the wrong sense. It was thus proposed to call this enthalpy retarded growth [43]. In the way of an example at the opposite extreme, the catabolism of autotrophic methanogenesis leads to a reduction of chemical entropy in the medium, which has to be overcome by an overly negative enthalpic driving force. These growth processes are therefore very exothermic [52] and could be called entropy retarded.

The question arises whether one can predict the way in which the driving force will be split up into an enthalpic and an entropic part. A relationship between $\Delta_{r} H_{\mathrm{X}}$ and $\Delta_{r} G_{\mathrm{X}}$ may be derived by eliminating $Y_{\mathrm{X} / \mathrm{S}}$ from Eqs. (20a) and (20b). The result is

$$
\frac{\Delta_{r} H_{\mathrm{X}}^{0}}{\Delta_{r} G_{\mathrm{X}}^{0}}=\frac{\Delta H_{\mathrm{a}}^{0}}{\Delta G_{\mathrm{a}}^{0}}\left[1-\frac{\Delta G_{\mathrm{b}}^{0}}{\Delta_{r} G_{\mathrm{X}}^{0}}\right]+\frac{\Delta H_{\mathrm{b}}^{0}}{\Delta_{r} G_{\mathrm{X}}^{0}} .
$$

A similar relationship has been developed by Heijnen et al. [47]. In aerobic growth, $\Delta H_{\mathrm{a}}^{0} \approx \Delta G_{\mathrm{a}}^{0}$ and $\Delta G_{\mathrm{b}}^{0} \approx \Delta H_{\mathrm{b}}^{0}$. In anaerobic growth, both $\Delta G_{\mathrm{b}}^{0}$

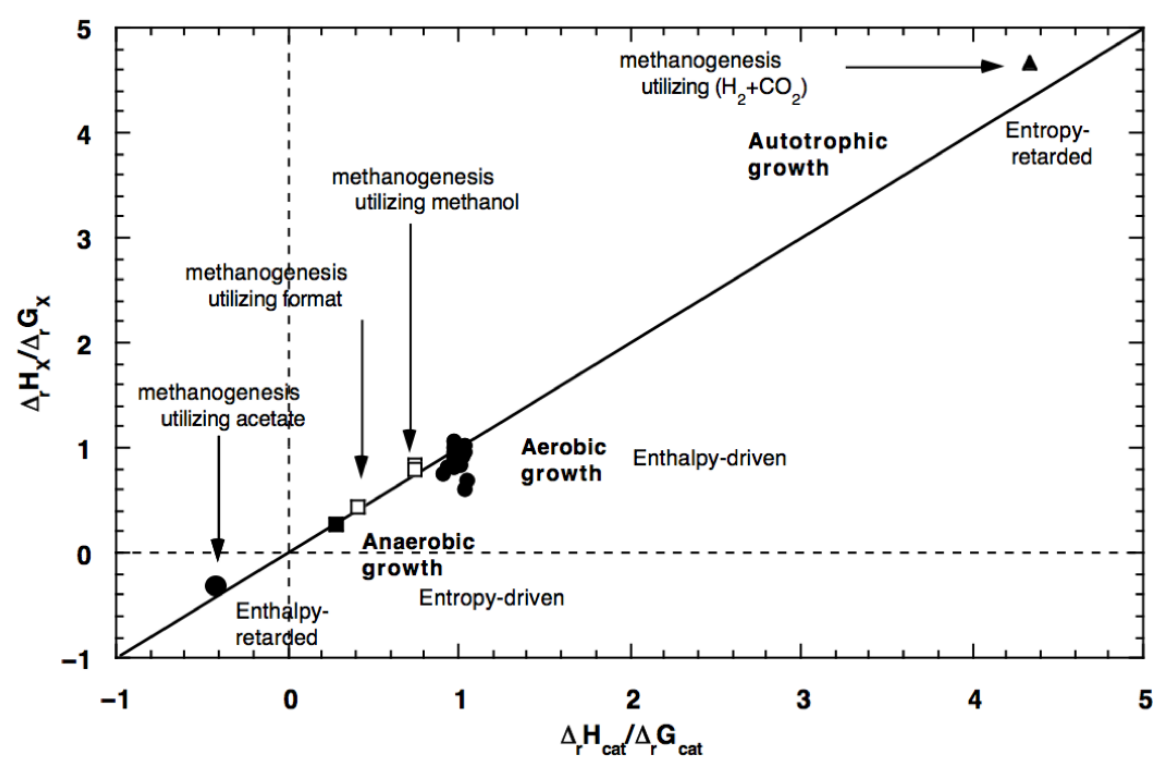

Figure 18. Plot of $\Delta_{r} H_{\mathrm{X}}^{0} / \Delta_{r} G_{\mathrm{X}}^{0}$ against $\Delta H_{\text {cat }}^{0} / \Delta G_{\text {cat }}^{0}$ for aerobic and anaerobic growth. Open keys: $\Delta_{r} H_{\mathrm{X}}^{0}$ were calculated based on an enthalpy balance; solid keys: $\Delta_{r} H_{\mathrm{X}}^{0}$ were measured calorimetrically. Adapted from von Stockar and Liu [43]. 
and $\Delta H_{\mathrm{b}}^{0}$ are small compared to $\Delta H_{\mathrm{b}}^{0}$. The result is in both cases

$$
\frac{\Delta_{r} H_{\mathrm{X}}^{0}}{\Delta_{r} G_{\mathrm{X}}^{0}} \approx \frac{\Delta H_{\mathrm{a}}^{0}}{\Delta G_{\mathrm{a}}^{0}}=1+\frac{T \Delta S_{\mathrm{a}}^{0}}{\Delta G_{\mathrm{a}}^{0}} .
$$

Equation (26) shows that the ratio of the enthalpy change and the Gibbs energy change accompanying growth ought to be approximately equal to the same ratio for the catabolic reaction alone.

This prediction is tested in Figure 18 by plotting the ratio of calorimetrically measured enthalpy changes and Gibbs energy changes calculated for the observed growth stoichiometry by Eq. (20a) versus $\Delta H^{0} / \Delta G^{0}$ for catabolism. The latter ratio may readily be estimated based on thermodynamic tables. Despite variations from -1 to over 4 in the latter ratio the correlation holds surprisingly well. Therefore, if $\Delta_{r} G_{\mathrm{X}}^{0}$ can be estimated (see below), the heat dissipation $\Delta_{r} H_{\mathrm{X}}^{0}$ may also be predicted by multiplying the former with $\Delta H^{0} / \Delta G^{0}$ for catabolism alone.

\section{Application: Prediction of the biomass yield}

Biomass or growth yield in cellular growth constitutes one of the key parameters in any scientific research project involving cellular cultures, since it determines the final biomass or cell concentration that may be obtained. It must be optimized imperatively in any biotechnological project, in order to produce reasonable amounts of biological material for scientific analysis and research. Optimizing the biomass yield is also of prime importance in industrial biotechnology for obtaining large product amounts and synthesis rates, both of which determine the economic viability of any bioprocess [3].

In view of its dominating importance among the parameters that characterize the growth behavior of cellular cultures, the prediction of biomass yield will serve as an example for the usefulness of thermodynamics as applied to biotechnology utilizing live cultures. The discussion will be limited to microbial cells because of the difficulties in analyzing the complexities of animal and other non-microbial cells. Even so, the challenge is considerable since growth yields for different microbial strains vary even under optimal conditions by about two orders of magnitude from less than $1 \%$ to more than $80 \%$ of C-mole dry biomass grown per C-mole of energy source consumed (for a graphic representation, see von Stockar et al. [3]). Biothermodynamics is expected to explain this variation and to yield a rational basis for correlating the data. 


\subsection{Prediction based on growth efficiency analysis and irreversible thermodynamics}

When developing a thermodynamic analysis for predicting biomass yields, it would seem natural to translate a large amount of experimentally measured growth yields into thermodynamic efficiency factors and to search for a way to correlate these. To this effect, many different bioenergetic efficiency coefficients have been defined [40,53-56], but it has been shown that many of these boil down to a so-called energy transducer efficiency characterizing the fraction of the Gibbs energy released by the driving reaction (a) that can be recovered in the form of Gibbs energy stored in the newly grown biomass [57]:

$$
\eta=-\frac{Y_{\mathrm{X} / \mathrm{S}} \Delta G_{\mathrm{b}}^{0}}{\Delta G_{\mathrm{a}}^{0}} .
$$

The most rigorous basis for developing a correlation for or even predicting thermodynamic efficiency factors is probably the linear energy transducer theory developed by Kedem and Caplan [58], Caplan and Essig [54], and Stucki [59] and reviewed by Gnaiger [60] and Westerhoff [61]. This theoretical development may be used to link practically important culture characteristics such as $Y_{\mathrm{X} / \mathrm{S}}$ and $\eta$ to fundamental parameters of irreversible thermodynamics. Although it is not possible to predict these parameters from first principles, and despite the fact that it is difficult to foresee whether such parameters are constant at all and under what circumstances, the theory can still be expected to yield some insight and predictions concerning the most probable or optimal values of the biotechnological characteristics.

The linear energy transducer theory is based on the assumption that the optimal bioenergetic efficiency would be the one affording the highest growth rate. In order to compute the growth rate as a function of the bioenergetic efficiency, the rate at which the catabolic reaction $r_{\mathrm{a}}$ is proceeding is first modeled according to the principles of irreversible thermodynamics:

$$
r_{\mathrm{a}}=L_{\mathrm{aa}} \cdot\left(-\Delta G_{\mathrm{a}}\right)+L_{\mathrm{ab}} \cdot\left(-\Delta G_{\mathrm{b}}\right) .
$$

$L_{\mathrm{aa}}$ and $L_{\mathrm{ab}}$ denote the phenomenological coefficients linking the rate of reaction to its conjugate driving force and to the force exerted by the biomass synthesis process, respectively. ( $L_{\mathrm{ab}}$ is negative and slows catabolism down, but the same coefficient also appears in a similar equation for $r_{\mathrm{b}}$ and ensures that this rate is positive and that the reaction runs uphill against the positive Gibbs energy of reaction.) 
If the two reactions are completely coupled and no "slip" exists, $L_{\mathrm{ab}}$ may be replaced [62] by $-\left(L_{\mathrm{aa}} \cdot L_{\mathrm{bb}}\right)^{1 / 2}$. In addition, it can be shown that the biomass yield is linked to the phenomenological coefficients as follows [3]:

$$
Y_{\mathrm{X} / \mathrm{S}}=\sqrt{\frac{L_{\mathrm{bb}}}{L_{\mathrm{aa}}}} .
$$

Substituting these two expressions into Eq. (28) yields $r_{\mathrm{a}}$ as a function of the biomass yield.

Since the reactions (a) and (b) have been defined as shown in Figure 11 assuming that the energy source is first completely catabolized and some of the catabolic products are then used to synthesize biomass, the rate of biomass synthesis $r_{\mathrm{b}}$ may subsequently be computed from the rate of catabolism as follows:

$$
r_{\mathrm{b}}=Y_{\mathrm{X} / \mathrm{S}} \cdot r_{\mathrm{a}}
$$

The result is

$$
r_{\mathrm{b}}=L_{\mathrm{aa}} \cdot\left(-\Delta G_{\mathrm{a}}^{0}\right) \cdot\left[Y_{\mathrm{X} / \mathrm{S}}-Y_{\mathrm{X} / \mathrm{S}}^{2} \frac{\Delta G_{\mathrm{b}}^{0}}{\Delta G_{\mathrm{a}}^{0}}\right] .
$$

This expression already shows that the rate of biomass synthesis goes through a maximum. However, the maximum may be seen more clearly when expressing $r_{\mathrm{b}}$ in terms of power $P=-r_{\mathrm{b}} \Delta G_{\mathrm{b}}^{0}$, i.e., the rate at which Gibbs energy is recovered in the newly synthesized biomass. If in addition $Y_{\mathrm{X} / \mathrm{S}}$ is substituted by $\eta$ by using Eq. (27), one obtains

$$
P=L_{\mathrm{aa}} \Delta G_{\mathrm{a}}^{2} \cdot \eta \cdot(1-\eta) .
$$

This expression may intuitively be understood. The power of Gibbs energy incorporation in new biomass must at the same time be proportional to the fraction of Gibbs energy retained in the biomass, i.e., to $\eta$, as well as to the growth rate, which in turn is proportional to the fraction of Gibbs energy dissipated and hence to $1-\eta$. A plot of this function would show a maximum at $\eta=0.5$. This illustrates quite well the compromise that must be stricken: both at $\eta=0$ and at $\eta=1$ the power becomes zero, and the maximum is found at an efficiency of $50 \%$.

In order to test several different concepts for correlating and predicting the biomass yield, Liu et al. [62] assembled a database of measured microbial biomass yields with over 200 different data points. This database was also used to test the prediction that the bioenergetic efficiency ought to amount approximately to $50 \%$ based on the linear energy transducer concept. As can be seen on Figure 19a, this prediction holds in aerobic cultures 
a)

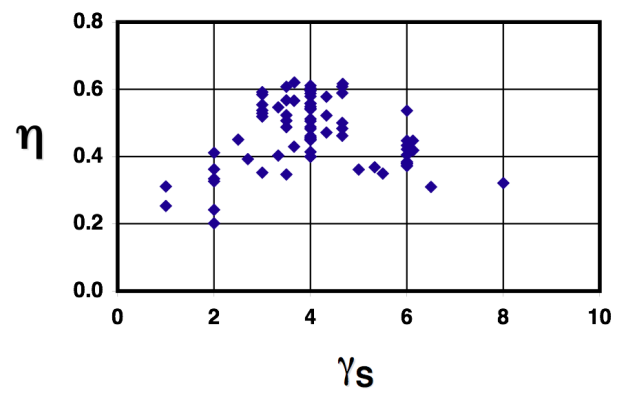

b)

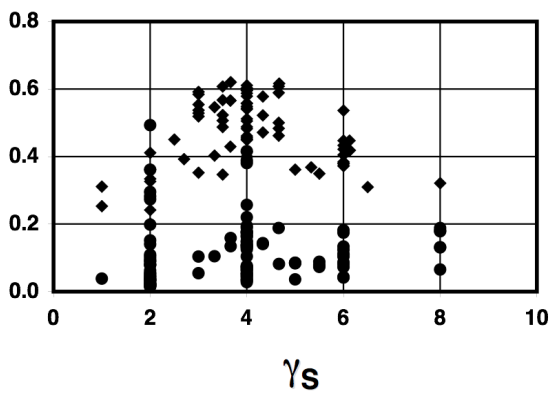

Figure 19. Bioenergetic efficiency as a function of the degree of reduction for a large number of literature results. (a) aerobic growth alone; (b) all results. Key: diamonds, aerobic growth; solid dots, anaerobic growth.

more or less. Most of the bioenergetic efficiency values varied from 0.4 to 0.6 for the stains investigated [3], and several literature reports of a wide variety of experimental data confirm that the thermodynamic efficiency often cluster around $50 \%$ (see, e.g., [3]).

However, the theory fails completely to predict the thermodynamic efficiency of anaerobic cultures. It was known already for a long time that these are much lower, often only amounting to a couple of percent or less. As shown in Figure 19b the bioenergetic efficiencies of aerobic and anaerobic growth taken together vary in an unpredictable way from above $60 \%$ down to close to $0 \%$. Moreover, Heijnen and van Dijken already pointed out in 1992, [47] and 1993, [63] that the concept of thermodynamic efficiency is plagued with serious internal inconsistencies. Their values indeed depend on exactly how the biosynthetic reaction is formulated. If Figure 10 is used to define them rather than Figure 11, one obtains much smaller values for $\Delta G_{\mathrm{b}}^{0}$, which in a considerable number of cases even turn out to be negative. Consequently, the thermodynamic growth efficiencies, too, become very small and in many cases negative (for data see, e.g., [3]). As compelling as the energy converter model may seem, it is not able to predict meaningful optimal growth efficiencies.

\subsection{Prediction based on energy transfer coefficients}

One of the earliest methods to predict microbial biomass yields for environmental applications was presented in 1969 by McCarty [64]. It was later considerably refined $[65,66]$ and finally resulted in the so-called Thermo- 
dynamic Electron Equivalents Model (TEEM [67,68]). Biomass synthesis, catabolic reactions, and reaction Gibbs energies are systematically formulated per electron equivalent. The method otherwise resembles the analysis presented in Section 4.1 in that the prediction is based on an empirical average value of an energy transfer coefficient $\varepsilon$ defined in quite an analogous way to $\eta$ (Eq. (27)):

$$
\varepsilon=-\frac{f_{\mathrm{S}}^{0}}{1-f_{\mathrm{S}}^{0}} \cdot \frac{\Delta G_{\mathrm{S}}}{\Delta G_{r}},
$$

where $f_{\mathrm{S}}^{0}$ is the fraction of the electrons in the energy substrate that end up in the new biomass, and $\Delta G_{\mathrm{S}}$ and $\Delta G_{r}$ stand for the reaction Gibbs energy of the biomass synthesis and the catabolic reaction, respectively.

$\varepsilon$ was found to be more or less close to 0.37 in many heterotrophic aerobic growth processes. Therefore, $f_{\mathrm{S}}^{0}$ may be predicted by solving Eq. (33) for it and subsequently transformed into a $\mathrm{C}$-molar biomass yield as follows:

$$
Y_{\mathrm{X} / \mathrm{S}}=f_{\mathrm{S}}^{0} \frac{\gamma_{\mathrm{S}}}{\gamma_{\mathrm{X}}}
$$

There is no clear reason why the energy transfer coefficient should adopt a constant value. The Second Law only places an upper bound of unity upon $\varepsilon$. The concept also suffers from the problem that $\varepsilon$ like $\eta$ adopts different values according to the formulation of the biosynthetic reactions. However, McCarty [68] proposes to assume that the carbon source is first transformed into Acetyl-CoA and provides an estimate of $\Delta \mathrm{G}$ of biomass synthesis from this intermediate based on the reported value of the ATP requirement.

The method was found to yield erroneous values for growth involving oxygenases and $\mathrm{C}_{1}$ carbon and energy substrates, and was subsequently modified and improved for these cases [69-71].

The yield predictions obtained by this method have been shown by van Briesen [72] to be comparable to those obtained from another well-established method (see next section) for oxidative organo-heterotrophic growth. However, the reliability and the accuracy of this method do not seem to have been tested systematically for lithothrophic, autotrophic, or anaerobic growth.

\subsection{Gibbs energy dissipation correlations}

As opposed to bioenergetic or the energy transfer efficiencies, the value of the Gibbs energy of the growth reaction $\Delta_{r} G_{\mathrm{X}}^{0}$ does not depend on how the biosynthetic reaction is formulated. This prompted Heijnen and van 
Dijken to search for a correlation for $\Delta_{r} G_{\mathrm{X}}^{0}$ rather than an efficiency measure as a basis for the prediction of the growth yield. Such a correlation was expected to be particularly simple to use because no assumptions on metabolic details would be required.

The compromise between the two unfavorable extreme situations that may have emerged during evolution (cf. Section 2.2.3) does perhaps indeed not involve an optimum energetic growth efficiency, but rather a certain amount of dissipated Gibbs energy. What might have happened during evolution is shown in Figure 20. Because of marked differences in $\Delta G_{\text {cat }}$ and $\Delta G_{\text {an }}$ in different microorganisms, the relation between biomass yield and Gibbs energy dissipation as indicated by Equation (20a) are numerically rather different from one type of catabolism to another (Figure 20). During evolution, however, an optimal amount of Gibbs energy change ranging in many cases perhaps from -300 to $-400 \mathrm{~kJ}$ per C-mole might have emerged. In each habitat, microorganisms dissipating more than this amount might have been outgrown by competitors with higher biomass yields, whereas other organisms with a lesser Gibbs energy dissipation might have been outgrown by competitors who grew faster.

Therefore different species of microorganisms might have adjusted their biomass yield in such a way that their Gibbs energy dissipation would fall more or less into that range.

If such a general rule permitting to estimate $\Delta_{r} G_{X}^{0}$ actually exists, the biomass yield can be estimated by solving Eq. (20a) for it:

$$
Y_{\mathrm{X} / \mathrm{S}}=\frac{\Delta G_{\mathrm{a}}^{0}}{\Delta_{r} G_{\mathrm{X}}^{0}-\Delta G_{\mathrm{b}}^{0}} .
$$

In a search for such a rule for use in Eq. (35), Heijnen and van Dijken [47, 63] examined $\Delta_{\mathrm{r}} G_{\mathrm{X}}^{0}$ values computed from a large literature basis and found that it is not really constant but depends on the degree of reduction and on the number of carbon atoms of the carbon source. They proposed the following empirical correlation:

$$
\begin{aligned}
-\Delta_{r} G_{\mathrm{X}}^{0}= & 200+18 \cdot\left(6-n_{\mathrm{C}}\right)^{1.8} \\
& +\exp \left[\left\{\left(3.8-\gamma_{\mathrm{C}}\right)^{2}\right\}^{0.16} \cdot\left(3.6+0.4 \cdot \mathrm{n}_{\mathrm{C}}\right)\right](\mathrm{kJ} / \mathrm{C}-\mathrm{mol}) .
\end{aligned}
$$

For growth with reverse electron transport they proposed:

$$
\Delta_{r} G_{\mathrm{X}}^{0}=-3500(\mathrm{~kJ} / \mathrm{C}-\mathrm{mol}) .
$$

The coefficients have no physical significance. However, by inspecting Eq. (36a) one finds that the Gibbs energy dissipation is minimal for carbon substrates of a degree of reduction of about 3.8 and of a number of 


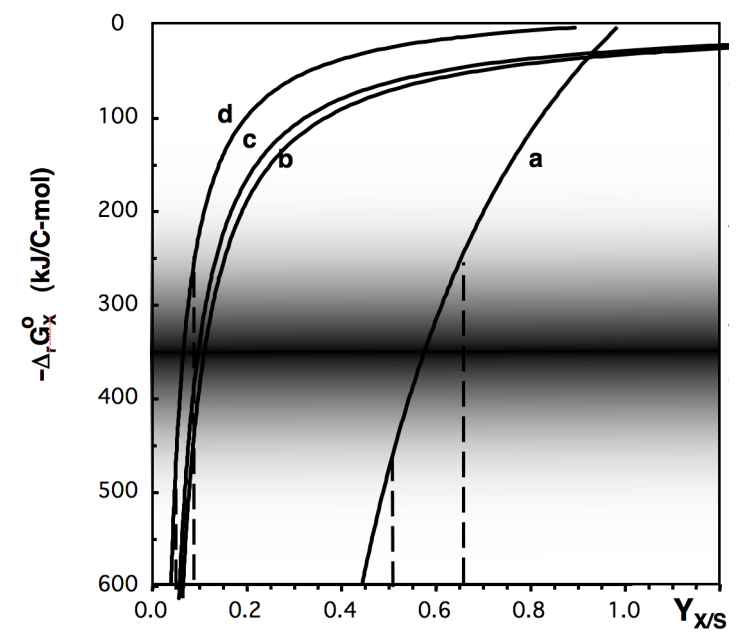

Figure 20. Gibbs energy of reaction calculated as a function of biomass yields from Eq. (20a) for (a) aerobic growth on glucose, (b) anaerobic ethanol fermentation on glucose, (c) lactic acid homofermentative growth on glucose, (d) acetotrophic methanogenesis. The shaded area may represent a favorable compromise between high growth efficiency and high growth rate. Pairs of broken lines: range of biomass yields for aerobic and acetotrophic methanogenetic growth. Adapted from [3].

carbon atoms of about 3. Organisms growing on carbon sources which have a different degree of reduction and/or a number of carbon atoms apparently tend to dissipate considerably more Gibbs energy. This has been explained $[47,63]$ by assuming that biomass is produced from precursors which have a degree of reduction of 3.8 and 3 carbon atoms. Growth on a substrate with these characteristics is therefore the easiest way to synthesize biomass from a biochemical point of view and requires the least amount of driving force. However, organisms that grow on carbon substrates that markedly differ from these values will have to first transform these into the precursor molecules. This needs more biochemical steps, and therefore more Gibbs energy must be dissipated for driving the added metabolic transformations.

More recently, a much simpler correlation was proposed as follows [62]:

$$
\begin{array}{ll}
\Delta_{r} G_{\mathrm{X}}^{0}=\frac{666.2}{\gamma_{\mathrm{S}}}+243.1(\mathrm{~kJ} / \mathrm{C}-\mathrm{mol}) & \text { for } \gamma_{\mathrm{S}} \leq 4.67, \\
\Delta_{r} G_{\mathrm{X}}^{0}=157 \gamma_{\mathrm{S}}-339(\mathrm{~kJ} / \mathrm{C}-\mathrm{mol}) & \text { for } \gamma_{\mathrm{S}}>4.67,
\end{array}
$$

where $\gamma_{S}$ denotes the degree of reduction of the energy source. 
Both Eqs. (36) and (37) were used in Eq. (35) in order to compare their ability to predict the biomass yields of a database containing 205 literature values including, among others, the whole database used earlier by Heijnen and van Dijken. It turned out that Eq. (37) predicted the biomass yield of reaction with a similar standard error of prediction of about $8 \%$ as Eq. (36) despite its dramatically simpler form. Even when $\Delta_{r} G_{\mathrm{X}}^{0}$ was simply assumed to be approximately constant such as suggested by Figure 20 and a value of $-500 \mathrm{~kJ} / \mathrm{C}$-mol was used in Eq. (35), the biomass yields were still predicted to about plus or minus $11 \%$ [62].

\subsection{Predictions based on ATP balances}

Early attempts to correlate the biomass yields were not based on thermodynamics, but rather on ATP balances [73-75]. The general idea was to estimate the amount of ATP yielded by catabolic reaction, and to compare the number with the so-called $\mathrm{Y}_{\mathrm{ATP}}$, which reflects the amount of ATP required to biosynthesize a unit of dry biomass. However, the method requires quite a large amount of metabolic information in order to estimate these ATP amounts and is thus not that useful for a yield prediction of novel, unknown strains. Since the concept is not based on thermodynamics, it will not be further discussed here.

\section{Thermodynamic analysis of culture parameters other than growth yield}

\subsection{Product and energy yields}

Product yields may also be estimated as long as the product results from catabolism. As it may be expected that the use of biofuels will increase dramatically in the near future and that even a growing fraction of bulk chemicals will be produced in a sustainable way in biorefineries, thermodynamic analyses of product formation and of the fraction of the energy retained in biofuels may become of interest for comparing different process options. Such processes will undoubtedly make use of the potential of anaerobic microorganisms to synthesize vast amounts of catabolic products from renewable resources. An actual analysis of this type is beyond the scope of this contribution, but the following lines are intended to be a very simple sketch of how such analyses could be performed.

The product yield $\mathrm{Y}_{\mathrm{P} / \mathrm{S}}$ may easily be estimated by multiplying the basic microbial growth stoichiometry shown in Eq. (19) by $Y_{\mathrm{X} / \mathrm{S}}$. The product 
yield will then appear as the stoichiometric coefficient of P. By performing a degree of reduction balance one obtains

$$
Y_{\mathrm{P} / \mathrm{S}}=\frac{\gamma_{\mathrm{S}}}{\gamma_{\mathrm{P}}}-\frac{\gamma_{\mathrm{X}}}{\gamma_{\mathrm{P}}} \cdot Y_{\mathrm{X} / \mathrm{S}}
$$

Estimating the biomass yield as described in the previous section using Eqs. (35) and (17) yields:

$$
Y_{\mathrm{P} / \mathrm{S}}=\frac{\gamma_{\mathrm{S}}}{\gamma_{\mathrm{P}}}-\frac{\gamma_{\mathrm{X}}}{\gamma_{\mathrm{P}}} \cdot \frac{\Delta_{\mathrm{X}} \mathrm{G}_{\mathrm{S}}^{0}-\gamma_{\mathrm{S}} \cdot \frac{\Delta_{\mathrm{C}} G_{\mathrm{P}}^{0}}{\gamma_{\mathrm{P}}}}{\Delta_{r} \mathrm{G}_{\mathrm{X}}^{0}-\gamma_{\mathrm{X}} \frac{\Delta_{\mathrm{c}} G_{\mathrm{P}}^{0}}{\gamma_{\mathrm{P}}}+\Delta_{r} \mathrm{G}_{\mathrm{P}}^{0}}
$$

The first term on the right-hand side of this equation indicates the theoretical maximum yield that could be obtained if one could work with solely uncoupled catabolism. Since in real processes vigorous growth might often also be necessary, the real yield is lower by the second term on the right, because some of the carbon source will be used for growing biomass. (In advanced processing options, this loss might however be minimized by working with immobilized biomass.)

The two values for the theoretical maximum yield and for the real yield with full microbial growth are plotted in Figure 21 for the production of different bulk chemicals from a renewable substrate that has been assumed to have the characteristics of a carbohydrate $\left(\mathrm{CH}_{2} \mathrm{O}\right)$. It may be seen that the

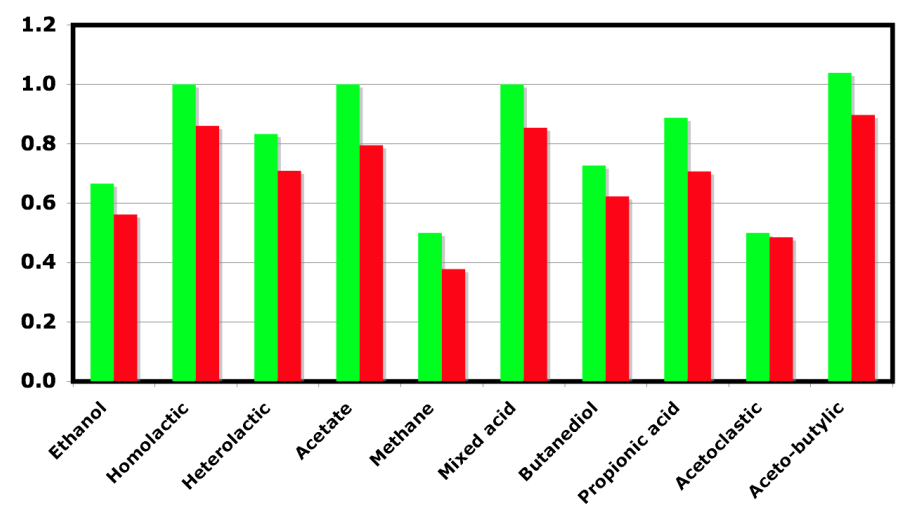

Figure 21. Theoretical (left columns) and real (right columns) product yields for several types of fermentations starting form carbohydrate as energy donor. The acetoclastic methanogenesis consumes acetate and not carbohydrate as substrate. 


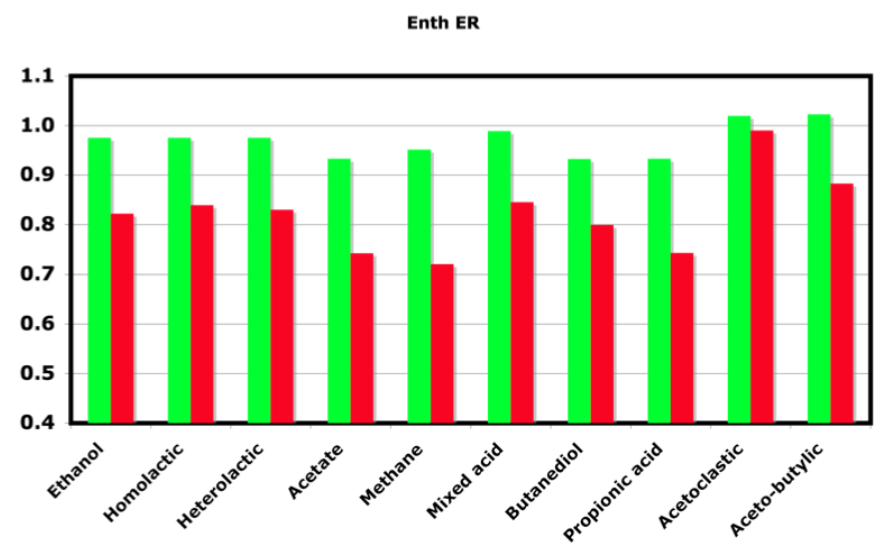

Gibbs ER

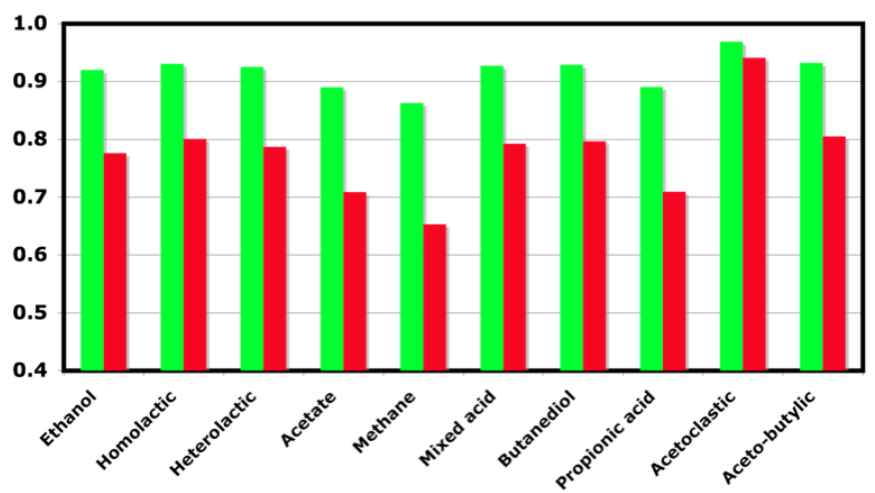

Figure 22. Energy recovery fraction for enthalpy (Enth ER) and Gibbs energy (Gibbs ER) that would be possible in different types of fermentations. Significance of columns and starting material like that in Figure 21.

yields differ quite substantially and may reach values approaching $100 \%$. Bioethanol reaches only an average yield and would be outperformed by some of the other biochemicals.

Figure 22 shows the enthalpic and Gibbs energy recoveries, respectively, that could be obtained with different products. This would be of importance if these fermentations would be used to produce biofuels according to different process variants. They have been calculated by multiplying the product yields by the energy content of the product and by dividing by the one of the carbon and energy substrate. It may be seen that the recovery 
fractions do not vary as widely as the product yields themselves. This is due to the fact that degrees of reduction are now replaced in the calculations with the ratios of degrees of reduction divided by the corresponding heat or Gibbs energy of combustion. It is widely known that these ratios are much more constant from one compound to another than the degrees of reduction themselves (cf. Eq. (7)).

Acetotrophic methanogenesis and acetobutylic fermentations reach very high recovery fractions, with theoretical values even exceeding unity. This is an indication that the fermentation may be endothermic, which has indeed been confirmed for the former case. Gibbs energy recovery cannot reach unity, because the Gibbs energy of reaction can be neither zero nor positive.

\subsection{Thermodynamic analysis of maintenance requirements and threshold concentrations}

During the 1990s, Heijnen and coworkers undertook thermodynamic analyses of a number of further essential parameters characterizing cellular growth. For instance, the correlations for the biomass yield discussed so far do not allow for the decrease of this parameter observed at low growth rates due to maintenance reactions. A complete prediction of the growth stoichiometry thus requires a correlation for estimating the maintenance coefficient, which then enables one to compute $\mathrm{Y}_{\mathrm{X} / \mathrm{S}}$ at low specific growth rates using the well-known Herbert-Pirt relation. Tijhuis et al. [76] placed this argument on a thermodynamic foundation by observing that maintenance reactions will increase the Gibbs energy dissipation rate as shown by Eq. (40):

$$
\Delta_{r} G_{\mathrm{X}}^{0} \cdot \mu=\left(\Delta_{r} G_{\mathrm{X}}^{0}\right)_{\min } \cdot \mu-\left(\mathrm{T} \cdot \dot{S}_{\mathrm{P}}\right)_{\mathrm{m}} \cdot
$$

The left-hand side and the first term on the right-hand side of Eq. (40) represent the total and the minimal Gibbs energy dissipation, respectively. $\left(\Delta_{\mathrm{r}} G_{\mathrm{X}}^{0}\right)_{\min }$ may be calculated by using Eqs. (36) and (37). The last term of Eq. (40) describes the constant Gibbs energy dissipation due to maintenance in $\mathrm{kJ} \mathrm{h}^{-1} \mathrm{C}-\mathrm{mol}^{-1}$.

The following correlation for the maintenance Gibbs energy dissipation was developed by Tijhuis et al. [76] based on an analysis of a large body of published data:

$$
\left(T \dot{S}_{\mathrm{P}}\right)=4.5 \cdot \exp \left\{\frac{-E_{\mathrm{a}}}{R}\left(\frac{1}{\mathrm{~T}}-\frac{1}{298}\right)\right\},
$$


where the activation energy $E_{\mathrm{a}}$ amounts to $69 \mathrm{~kJ} / \mathrm{mol}$. According to the authors, Eq. (41) reproduces the published data to within a standard deviation between $40 \%$ and $50 \%$.

Heijnen [77] also presents correlations for a number of kinetic growth parameters. On the basis of the assumption that the liberation of Gibbs energy in the electron transport chain is the growth limiting phenomenon, an expression for $\mu_{\max }$ was developed. He also developed thermodynamic arguments for calculating the residual substrate concentration $\mathrm{c}_{\min }$ at which growth ceases due to maintenance requirements and the threshold substrate concentration $c_{\text {thresh }}$ at which no more substrate can be taken up because the driving catabolic reaction and the ATP generating system (e.g., the proton motive force) are in equilibrium. While it is clear that these arguments yield plausible values, the prediction methods have not been widely tested and their accuracy is not known.

\section{Conclusions}

Live organisms must constantly dissipate Gibbs energy. In chemotrophic microbes, this dissipation reflects directly the export of excess entropy produced by all the irreversible processes associated with life functions. Without continuous export, entropy produced in the cells would tend to accumulate, thereby increasing its entropy content and thus would lead to a breakdown of the highly organized structures existing in live biomass. This problem is compounded in growing cells by the fact that they must synthesize new biomass, which is characterized by an entropy content as low or even lower than the one of the nutritional substrates, which have been assimilated by the cells in order to construct the new biomass.

It is the role of the catabolic reactions to assure this entropy export. Catabolism either converts some of the growth substrates into waste products of much lower energy content, thereby generating and exporting heat, which represents one form of entropy export. This type of catabolism will be accompanied by a strongly negative enthalpy change $\Delta_{r} H$, which can be experimentally measured in a calorimeter. The ubiquity of the export of entropy in the form of heat explains why almost all living organisms dissipate heat. Yet another possibility, mainly seen in fermentations, is the disintegration of substrate molecules into smaller ones, which thus contain more entropy than the substrates and can be wasted into the environment with a net loss of entropy for the cells. The export is thus accompanied by an increase of chemical entropy in the medium, i.e., by a positive $\Delta_{r} S$. As 
has been experimentally demonstrated at least for one type of fermentation, $\Delta_{r} S$ may become so large that it may overcompensate for a positive $\Delta_{r} H$, thus enabling the existence of endothermic life forms.

Because of the fact that the negative Gibbs energy change of growth directly reflects the entropy export and thus the entropy production in chemotrophic cells, it may be regarded as the driving force for growth. According to non-equilibrium thermodynamics, the more entropy can be produced during growth, i.e., the more $\Delta_{r} G$ is negative, the faster metabolic processes tend to run. As the principal contributor to a negative $\Delta_{r} G$ is catabolism, which does not produce any biomass, the driving force $\Delta_{r} G$ will be stronger and the growth faster, the smaller the biomass yield is. Cells must therefore strike a compromise between the extremes of ultrafast, wasteful metabolism with very low yields on the one hand, and very efficient but slow metabolism on the other hand. The analysis of a large amount of experimental data suggests the hypothesis that microorganisms might have reached this compromise by adapting their biomass yields in such a way that an "optimal" driving force $\Delta_{r} G$ ensues, which often lies between -250 and $-500 \mathrm{~kJ} / \mathrm{C}$-mol of dry biomass grown.

These concepts may be used in order to roughly predict important culture performance parameters. To this effect, a simple Gibbs energy balance over the cell is solved for the growth yield, but one needs an estimation of the amount of Gibbs energy the cells dissipate per unit of biomass grown. The literature suggests several methods to obtain such an estimate, including calculating it from empirical average values of an energy transfer efficiency, from theoretical predictions of the bioenergetic efficiency based on irreversible thermodynamics, or from direct empirical correlations of the "optimal" reaction Gibbs energy dissipation accompanying growth. The latter is particularly useful because it does not require any assumptions on the details of anabolism, which are usually unknown, and because it has been tested against a large amount of microbial growth data.

Employing the same concept, the catabolic product yield and the energy yield may be assessed, which might be of use for strategic planning of biorefineries and biofuel manufacture. Culture parameters such as specific growth rates, maintenance requirements, and threshold concentrations have also been shown to be amenable to Gibbs energy analysis.

However, the analysis reviewed in this contribution is essentially based on a black-box approach, in which a single parameter (e.g., biomass yield) will determine the entire stoichiometry. Although it may be extended to more complex cases if additional yield coefficients are known, more complicated phenomena such as yields of products that are unrelated to cata- 
bolism, stoichiometries in animal cell cultures, or the thermodynamic feasibility of metabolic engineering projects cannot be analyzed based on such a simple black-box model. To tackle them, a full-blown thermodynamic analysis of genome-wide metabolism would be needed.

\section{Acknowledgments}

I am indebted to a large number of former collaborators for having contributed to the experimental results described in this review. Among the most important are I. W. Marison, S. Ampuero, B. Birou, B. Butsch, J.-L. Cordier, P. Duboc, C. Jungo, C. Larsson, J.-S. Liu, T. Maskow, R. Patiño, P. Pugeaud, N. Schill, W. M. van Gulik, D. Voisard, and V. Vojinovic. I would like to thank E.H. Battley, E. Gnaiger, J. J. Heijnen, L. Gustafsson, J. U. Keller, R. B. Kemp, and J. M. Prausnitz for many stimulating discussions on biothermodynamics during all these years. I am grateful for long-term financial support from the Swiss National Science Foundation.

\section{List of symbols}

A Heat transfer area, $\mathrm{m}^{2}$;

A Electron acceptor

$\bar{c}_{\mathrm{P}} \quad$ Mean heat capacity of system, $\mathrm{kJ} \mathrm{kg}^{-1} \mathrm{~K}^{-1}$

$\Delta_{r} G_{i}^{0} \quad$ Standard Gibbs energy of reaction per mole of $i, \mathrm{~kJ} \mathrm{~mol}^{-1}$ or kJ C-mol ${ }^{-1}$

$\Delta_{\mathrm{c}} G_{i}^{0} \quad$ Standard Gibbs energy of combustion of $i, \mathrm{~kJ} \mathrm{~mol}^{-1}$ or $\mathrm{kJ} \mathrm{C}-\mathrm{mol}^{-1}$

$\Delta G_{\text {cat }}^{0}, \Delta G_{\mathrm{a}}^{0} \quad$ Standard Gibbs energy of catabolism, same units (Eq. (16a))

$\Delta G_{\text {bios }}^{0}, \Delta G_{\mathrm{b}}^{0} \quad$ Standard Gibbs energy of "biosynthesis" or "anabolism," same units (Eq. (16b))

$\Delta_{r} H_{i}^{0} \quad$ Standard molar enthalpy of reaction per mole of species $i$,

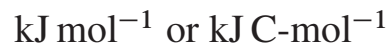

$\Delta_{\mathrm{c}} H_{i}^{0} \quad$ Standard enthalpy of combustion of $i, \mathrm{~kJ} \mathrm{~mol}^{-1}$ or $\mathrm{kJC}-\mathrm{mol}^{-1}$

$\Delta H_{\mathrm{a}}^{0}, \Delta H_{\mathrm{b}}^{0} \quad$ Standard molar enthalpy of reaction of "catabolism" and “anabolism," kJ mol${ }^{-1}$ or kJ C-mol ${ }^{-1}$ (Eq. (16)) 
$\bar{h}_{i}$

$\dot{n}_{i, e}$

$p$

$Q$

$\dot{Q}$

$\dot{q}$

$r_{j}$

$r_{\mathrm{S}}$

$r_{\mathrm{X}}$

$s$

$\dot{S}_{\text {prod }}$

$\Delta_{r} S_{i}^{0}$

$\bar{s}_{i}$

SOX

$T$

$T_{e}$

$T_{\mathrm{R}}$

$T_{\mathrm{J}}$

$U$

V

$\dot{W}$

$\mathrm{X}$

$x$

$x_{k}$

$Y_{i / j}$

$Y_{Q / \mathrm{X}}$
Partial molar enthalpy of $i$-th compound in the system

Flow rate of $i$-th species into system, $\mathrm{mol} \mathrm{s}^{-1}$

Pressure, $\mathrm{kPa}$

Total heat, kJ

Heat flux into the system, W

Heat flux exchanged between the system and the environment, $\mathrm{W} \mathrm{m}^{-3}$

Reaction rate of $j$-th reaction, $\mathrm{mol} \mathrm{h}^{-1} \mathrm{~m}^{-3}$

Rate of substrate consumption, $\mathrm{C}-\mathrm{mol} \mathrm{h}^{-1} \mathrm{~m}^{-3}$

Rate of growth, $\mathrm{C}-\mathrm{mol} \mathrm{h}^{-1} \mathrm{~m}^{-3}$

Concentration of carbon and energy substrate, $\mathrm{C}-\mathrm{mol} \mathrm{m}^{-3}$ or $\mathrm{mol} \mathrm{m}^{-3}$

Rate of entropy production, $\mathrm{kJ} \mathrm{K}^{-1} \mathrm{~s}^{-1}$

Standard entropy of reaction per mole or C-mole of $i$,

$\mathrm{kJ} \mathrm{K}^{-1} \mathrm{~mol}^{-1}$ or per mole or C-mole of $i, \mathrm{~kJ} \mathrm{~K}^{-1} \mathrm{C}-\mathrm{mol}^{-1}$

Partial molar entropy of $i, \mathrm{~kJ} \mathrm{~K}^{-1} \mathrm{~mol}^{-1}$ or $\mathrm{C}-\mathrm{mol}^{-1}$

Oxidized energy source

System temperature, K

Temperature of stream entering or leaving through the $e$-th exchange port

Reaction temperature, $\mathrm{K}$

Jacket temperature, $\mathrm{K}$

Overall transmission coefficient, $\mathrm{W} \mathrm{m}^{-2} \mathrm{~s}^{-1}$

Volume of system or culure, $\mathrm{m}^{3}$

Work supplied, W

Biomass

Biomass concentration, $\mathrm{C}-\mathrm{mol} \mathrm{m}{ }^{-3}$

Atomic coefficient in elemental biomass formula, with $k=\mathrm{H}$ for hydrogen, $k=\mathrm{O}$ for oxygen, $k=\mathrm{N}$ for nitrogen

Yield or stoichiometric coefficient of $i$ per $j$, mol C-mol ${ }^{-1}$ or C-mol C-mol ${ }^{-1}$

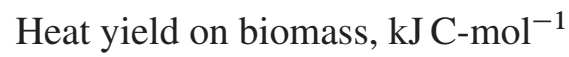




\begin{tabular}{|c|c|}
\hline $\mathrm{Y}_{\mathrm{X} / \mathrm{S}}$ & Biomass yield, C-mol C-mol ${ }^{-1}$ \\
\hline$\gamma_{i}^{0}$ & $\begin{array}{l}\text { Karash degree of reduction (using } \mathrm{CO}_{2}, \mathrm{H}_{2} \mathrm{O} \text {, and } \mathrm{N}_{2} \\
\text { as reference state). For a compound of atomic formula } \\
\mathrm{CH}_{x_{\mathrm{H}}} \mathrm{O}_{x_{\mathrm{O}}} \mathrm{N}_{x_{\mathrm{N}}}: \gamma_{i}^{0}=4+x_{\mathrm{H}}-2 x_{\mathrm{O}}\end{array}$ \\
\hline$\gamma_{i}$ & $\begin{array}{l}\text { Generalized degree of reduction, using } \mathrm{CO}_{2}, \mathrm{H}_{2} \mathrm{O} \text {, and } \\
\mathrm{NH}_{3} \text { as reference state, } \gamma_{i}^{0}=4+x_{\mathrm{H}}-2 x_{\mathrm{O}}\end{array}$ \\
\hline$\varepsilon$ & Energy transfer coefficient (Eq. (33)) \\
\hline$\mu_{i}$ & Chemical potential of $i$-th species \\
\hline$v_{i, j}$ & $\begin{array}{l}\text { Stoichiometry coefficient of } i \text {-th species in } \\
\text { the } j \text {-th reaction }\end{array}$ \\
\hline$\eta$ & Bioenergetic growth efficiency (Eq. (27)) \\
\hline$\rho$ & Density of system, $\mathrm{kg} \mathrm{m}^{-3}$ \\
\hline$\dot{\xi}_{j}$ & Absolute rate of reaction $j$, mol or $\mathrm{C}-\mathrm{mol} \mathrm{s}^{-1}$ \\
\hline
\end{tabular}

\section{Subscripts}
A Electron acceptor
$\mathrm{N} \quad$ Nitrogen source
O Oxygen
$\mathrm{P} \quad$ Product
$\mathrm{C} \quad \mathrm{CO}_{2}$
S Carbon and energy substrate, or energy substrate
SOX Oxidized energy source
$\mathrm{X}$ Biomass
W $\quad \mathrm{H}_{2} \mathrm{O}$
$i \quad i$-th chemical compound
$e \quad e$-th entry or exit port. Without this subscript: referring to system
$j \quad j$-th chemical reaction

\section{Superscripts}

a Pertaining to reaction (a) (Eq. (16))

b Pertaining to reaction (b) (Eq. (16)) 


\section{Bibliography}

[1] von Stockar, U. and van der Wielen, L. A. M., Back to basics, Thermodynamics in biochemical engineering, Adv. Biochem. Eng./Biotechnol., 80 (2003), $1-17$.

[2] von Stockar, U., Maskow, Th., Liu, J.-S., Marison, I. W. and Patiño, R., Thermodynamics of microbial growth and metabolism: An analysis of the current situation, J. Biotechnol., 121 (2006), 517-533.

[3] von Stockar, U., Vojinović, V., Maskow, Th. and Liu, J.-S., Can microbial growth yield be estimated using simple thermodynamic analogies to technical processes? Chem. Eng. Process., 47 (2008), 980-990.

[4] von Stockar, U., van der Wielen, L. A. M., Back to basics: thermodynamics in biochemical engineering, course binder, intensive course on thermodynamics in biochemical engineering, Biedenkopf, October 12-17, 2008, DECHEMA e.V. Frankfurt, 2008.

[5] von Stockar, U. and Marison, I. W., The use of calorimetry in biotechnology, Adv. Biochem. Eng. Biotechnol., 40 (1989), 93-136.

[6] von Stockar, U. and Marison, I. W., Large-scale calorimetry in biotechnology, Thermochim. Acta, 193 (1991), 215-242.

[7] Türker, M., Development of biocalorimetry as a technique for process monitoring and control in technical scale fermentations, Thermochim. Acta, 419 (2004), 73-81.

[8] Voisard, D., Pugeaud, P., Kumar, A. R., Jenny, K., Jayaraman, K., Marison, I. W. and von Stockar, U., Development of a large-scale biocalorimeter to monitor and control bioprocesses, Biotechnol. Bioeng., 80 (2002), 125-138.

[9] von Stockar, U., Gustafsson, L., Larsson, C., Marison, I. W., Tissot, P. and Gnaiger, E., Thermodynamic considerations in constructing energy balancs for cellular growth, Biochim. Biophys. Acta, 1183 (1993), 221-240.

[10] Ishikawa, Y., Nonoyama, Y. and Shoda, M., Microcalorimetric study of aerobic growth of $E$. coli in batch culture, Biotechnol. Bioeng., 23 (1981), 28252836.

[11] Larsson, C., Lidén, G., Niklasson, C. and Gustavsson, L., Calorimetric control of fed-batch cultures of Saccharomyces cerevisiae, Bioprocess Eng., 7 (1991a), 151-155. 
[12] Larsson, C., Blomberg, A. and Gustavsson, L., The use of microcalorimetric monitoring in establishing continuous energy balances and in continuous determinations of substrate and product concentrations of batch-growth Saccharomyces cerevisiae, Biotechnol. Bioeng., 38 (1991b), 447-458.

[13] Guan, Y. H., Lloyd, P. C. and Kemp, R. B., A calorimetric flow vessel optimised for measuring the metabolic activity of animal cells, Thermochim. Acta, 332 (1999), 211-220.

[14] Guan, Y. H. and Kemp, R. B., Detection of the changing substrate requirements of cultured animal cells by stoichiometric growth equations validated by enthalpy balances, J. Biotechnol. 69 (1999), 95-114.

[15] Kemp, R. B. and Guan, Y. H., The application of heat flux measurement to improve the growth of mammalian cells in culture, Thermochim. Acta, 332 (2000), 23-30.

[16] Maskow, Th., Olomolaye, D., Breuer, U., Kemp, R. B., Flow calorimetry and dielectric spectroscopy to control the bacterial conversion of toxic substrates into PHA, Biotechnol. Bioeng., 85 (2004), 547-552.

[17] Cooney, C. L., Wang, D. I. C. and Mateles, R. I., Measurement of heat evolution and correlation with oxygen consumption during microbial growth, Biotechnol. Bioeng., 11 (1968), 269-281.

[18] van Kleeff, B. H. A., Kuenen, J. G. and Heijnen, J. J., Continuous measurement of microbial heat production in laboratory fermentors, Biotechnol. Bioeng., 41 (1993), 541-549.

[19] van Kleeff, B. H. A., Kuenen, J. G., Honderd, G. and Heijnen, J. J., Model based optimization of equipment and control for heat flux measurements in a laboratory fermentor, Biotechnol. Prog., 11 (1995), 525-532.

[20] van Kleeff, B. H. A., Kuenen, J. G. and Heijnen, J. J., Heat flux measurements for the fast monitoring of dynamic responses to glucose additions by yeasts that were subjected to different feeding regimes in continuous culture, Biotechol. Prog., 12 (1996), 510-518.

[21] van Kleeff, B. H. A., Kuenen, J. G., Honderd, G. and Heijnen, J. J., Using heat-flow measurements for the feed control of a fed-batch fermentation of Saccharomyces cerevisiae, Thermochim. Acta, 309 (1998), 175-180.

[22] Meier-Schneiders, M. and Schäfer, F., Quantification of small enthalpic differences in anaerobic metabolism: A calorimetry supported approach, Thermochim. Acta, 275 (1996), 1-16. 
[23] Maskow, Th. and Babel, W., Calorimetric investigation of bacterial growth on phenol, Thermochim. Acta, 309 (1998), 97-103.

[24] Maskow, Th. and Babel, W., Calorimetrically recognized maximum yield of PHB continuously synthesized from toxic substrates, J. Biotechnol., 77 (2000), 247-253.

[25] Maskow, Th. and Babel, W., Calorimetrically obtained information about the efficiency of ectoine synthesis from glucose in Halomonas elongata, Biochim. Biophys. Acta, 1527 (2001), 4-10.

[26] Maskow, Th. and Babel, W., Calorimetric analysis of microorganisms in transient growth states to quantify changes of metabolic fluxes in response to nutrient deficiencies and osmostress, Thermochim. Acta, 382 (2002), 229-237.

[27] Marison, I. W. and von Stockar, U., A novel bench-scale calorimeter for biological process development work, Thermochim. Acta, 85 (1985), 497500 .

[28] Senthilkumar, S., Surianarayanan, M. and Swaminathan, G., Biocalorimetric and respirometric studies on biological treatment of tannery saline water, Appl. Microbiol. Biotechnol., 78 (2008), 249-255.

[29] Surianarayanan, M. and Senthilkumar, S., Bioenergetic studies on aerobic growth of Pseudomonas aeruginosa in a single substrate media, J. Chem. Technol. Biotechnol., 84 (2009), 1234-1239.

[30] Marison, I. W., Liu, J.-S., Ampuero, S., von Stockar, U. and Schenker, B., Biological reaction calorimetry: Development of high sensitivity calorimeter, Thermochim. Acta, 309 (1998), 157-153.

[31] Maskow, Th. and Harms, H., Real time insight into bioprocesses using calorimetry: State of the art and potential, Eng. Life Sci., 6 (2006), 266277.

[32] Maskow, Th., Müller, S., Lösche, A., Kemp, R. B. and Harms, H., Control of continuous polyhydroxyalkanoate synthesis using calorimetry and flow cytometry, Biotechnol. Bioeng., 93 (2006), 541-552.

[33] Lerchner, J., Maskow, Th. and Wolf, G., Chip calorimetry and its use for biochemical and cell biological investigations, Chem. Eng. Process., 47 (2008), 991-999.

[34] Lerchner, J., Wolf, A., Buchholz, F., Mertens, F., Neu, T. R., Harms, H. and Maskow, Th., Miniturized calorimetry - A new method for ereal biofilm activity analysis, J. Microbiol. Methods, 47 (2008), 74-81. 
[35] Birou, B., Marison, I. W. and von Stockar, U., Calorimetric investigation of aerobic fermentations, Biotechnol. Bioeng., 30 (1987), 650-660.

[36] Thornton, W. M., The relation of oxygen to the heat of combustion of organic compounds, Philos. Mag., 33 (1917), 196-203.

[37] Patel, S. A. and Erickson, L. E., Estimation of heat of combustion of biomass from elemental analysis using available electron concepts, Biotechnol. Bioeng., 23 (1981), 2051-2067.

[38] Cordier, J.-L., Butsch, B. M., Birou B. and von Stockar, U., Mini-review: The relationship between elemental composition and heat of combustion of microbial biomass, Appl. Microbiol. Biotechnol., 25 (1987), 305-312.

[39] Sandler S. I. and Orbey, H., On the thermodynamics of microbial growth processes, Biotechnol. Bioeng., 38 (1991), 697-718.

[40] Roels, J. A., Energetics and kinetics in biotechnology, Elsevier Biomedical Press, Amsterdam, 1983.

[41] Jungo, C., Marison, I. W. and von Stockar, U., Mixed feeds of glycerol and methanol can improve the performance of Pichia pastoris cultures: A quantitative study based on concentration gradients in transient continuous cultures, J. Biotechnol., 128 (2007), 824-837.

[42] Jungo, C., Schenk, J., Pasquier, M., Marison, I. W. and von Stockar, U., A quantitative analysis of the benefits of mixed feeds of sorbitol and methanol for the production of recombinant avidin with Pichia pastoris, J. Biotechnol., 131 (2007), 57-66.

[43] von Stockar, U. and Liu, J.-S., Does microbial life always feed on negative entropy? Thermodynamic analysis of microbial growth, Biochim. Biophys. Acta, 1412 (1999), 191-211.

[44] Duboc, P., Marison, I. W. and von Stockar, U., Quantitative calorimetry and biochemical engineering, in: Handbook of Thermal Analysis and Calorimetry; From Macromolecules to Man, Ed. R. B. Kemp, pp. 267-365, Elsevier, Amsterdam, 1999.

[45] Gürakan, T., Marison, I. W., von Stockar, U., Gustavsson, L. and Gnaiger, E., Proposals for a standardized sample handling procedure for the determination of elemental composition and enthalpy of combustion of biological material, Thermochim. Acta, 172 (1990), 251-266. 
[46] Battley, E. H., Putnam, R. L. and Boerio-Gates, J., Heat capacity measurements from 10 to $300 \mathrm{~K}$ and derived thermodynamic functions of lyophilized cells of Saccharomyces cerevisiae, including the absolute entropy and the entropy of formation at $298.15 \mathrm{~K}$, Thermochim. Acta, 298 (1997), 37-46.

[47] Heijnen, J. J. and van Dijken, J. A., In search of thermodynamic description of biomass yields for the chemotrophic growth of micro-organisms, Biotechnol. Bioeng., 39 (1992), 833-858.

[48] Liu, J. S., Marison, I. W. and von Stockar, U., Anaerobic calorimetry of the growth of Lactobacillus helveticus using a highly sensitive Bio-RC1, J. Therm. Anal. Calorimetry, 56 (1999), 1191-1195.

[49] Liu, J. S., Marison, I. W. and von Stockar, U., Microbial growth by a net heat up-take: A calorimetric and thermodynamic study on acetotrophic methanogenesis by Methanosarcina barkeri, Biotechnol. Bioeng., 75 (2001), 170180.

[50] Keller, J. U., An outlook to biothermodynamics. I. Biomolecules and proteins, J. Non-Equilib. Thermodyn., 33 (2008), 321-385.

[51] Schill, N., van Gulik, W. M., Voisard, D. and Von Stockar, U., Continuous cultures limited by a gaseous substrate: Development of a simple, unstructured mathematical model and experimental verification with Methanobacterium thermoautotrophicum, Biotechnol. Bioeng., 51 (1996), 645-658.

[52] Schill, N., Liu, J. S. and von Stockar, U., Thermodynamic analysis of growth of Methanobacterium thermoautotrophicum, Biotechnol. Bioeng., 64 (1999), 75-81.

[53] Atkinson, D. E., Cellular energy metabolism and its regulation, Academic Press, New York, 1977.

[54] Caplan, R. S. and Essig, A. Bioenergetics and linear non equilibrium thermodyamics: The steady state, Harvard University Press, Cambridge, MA, 1983.

[55] Cornish-Bowden, A. Metabolic efficiency: Is it a useful concept? Biochem. Soc. Trans., 11 (1983), 44-45.

[56] Battley, E. H., Energetics of Microbial Growth, J. Wiley, New York, 1987.

[57] von Stockar, U. and Marison, I. W., The definition of energetic growth efficiencies for aerobic and anaerobic microbial growth and their determination by calorimetry and other means, Thermochim. Acta, 229 (1993), 157-172. 
[58] Kedem, O. and Caplan, S. R., Degree of coupling and its relation to efficiency of energy conversion, Trans. Faraday Soc., 61 (1965), 1897-1911.

[59] Stucki, J. W., The optimal efficiency and economic degrees of coupling of oxidative phosphorylation, Eur. J. Biochem, 109 (1980), 269-283.

[60] Gnaiger, E., Concepts of efficiency in biological calorimetry and metabolic flux control, Thermochim. Acta, 172 (1990), 31-52.

[61] Westerhoff, H. V. and van Dam, K., Thermodynamics and Control of Biological Free-Energy Transduction, Elsevier, Amsterdam, 1987.

[62] Liu, J.-S., Vojinovic, V., Patino, R., Maskow, Th. and von Stockar, U., A comparison of various Gibbs energy dissipation correlations for predicting microbial growth yields, Thermochim. Acta, 458 (2007), 38-46.

[63] Heijnen, J. J. and van Dijken, J. P., Response to comments on: In search of a thermodynamic description of biomass yields for the chemotrophic growth of micro-organisms, Biotechnol. Bioeng., 42 (1993), 1127-1130.

[64] McCarty, P. L., Energetics and bacterial growth. in: S. D. Faust, J. V. Hunter (Eds.), Organic Compounds in Aqueous Environments, Marcel Dekker Inc., New York, 1971, 495-531.

[65] Lawrence, A. W. and McCarty, P. L., Unified Basis for biological treatment design and operation. ASCE J. Sanit. Eng. Div., 96 (1970), 757-778.

[66] Christensen, D. R. and McCarty, P. L., Multiprocess biological treatment model, J. Water Pollut. Control Fed., 47 (1975), 2652-2664.

[67] Rittmann, B. E. and McCarty, P. L., Environmental Biotechnology: Principles and Applications, McGraw-Hill, New York, 2001.

[68] McCarty, P. L., Thermodynamic electron equivalents model for bacterial yield prediction: Modifications and comparative evaluations, Biotechnol. Bioeng. 97 (2007), 377-388.

[69] Van Briesen, J. M., Thermodynamic yield predictions for biodegradation through oxygenase activation reactions, Biodegradation, 12 (2001), 265281.

[70] Yuan, Z. and Van Briesen, J. M., Yield prediction and stoichiometry of multistep biodegradation reactions involving oxygenation, Biotechnol. Bioeng., 80 (2002), 100-113.

[71] Xiao, J. and Van Briesen, J. M., Expanded thermodynamic model for microbial true yield prediction, Biotechnol. Bioeng., 93 (2006), 110-121. 
[72] Van Briesen, J. M., Evaluation of methods to predict bacterial yield using thermodynamics, Biodegradation, 13 (2002), 171-190.

[73] Beauchop, T. and Elsden, S. R., The growth of microorganisms in relation to their energy supply, J. Gen. Microbiol., 23 (1960), 457-469.

[74] Stouthamer, A. H., In search of a correlation between theoretical and experimental growth yields, in: Microbial Biochemistry, vol. 21, Ed. J. R. Quayle, pp. 1-48, University Park Press, Baltimore, 1979.

[75] Maskow, Th., Thermodynamische Charakterisierung und kalorimetrische Steuerung mikrobieller Leistungen in Uebergangssituationen, Habilitationsschrift, Universität Dresden, 2005.

[76] Tijhuis, L., van Loosdrecht, M. and Heijnen, J. J., A Thermodynamically based correlation for maintenance Gibbs energy requirements in aerobic and anaerobic chemotrophic growth, Biotechnol. Bioeng., 42 (1993), 509-519.

[77] Heijnen, J. J., Bioenergetics of microbial growth, in: Bioprocess Technology: Fermentation, Biocatalysis and Bioseparation, Eds. M. C. Flickiger and S. W. Drew, pp. 267-291, J. Wiley \& Sons, Chicester UK, 1999.

[78] Frenz, U., Whole body calorimetry, in: Handbook of Thermal Analysis and Calorimetry, vol. 4, From Macromolecules to Man, Ed. R. B. Kemp, pp. 511-555, Elsevier SA, Amsterdam, 1999.

[79] Lavoisier, A. L. and de Laplace, P. S., Mémoire sur la chaleur, Histoire de l'Académie des Sciences, p. 355, Académie des Sciences, Paris, 1780.

[80] Lamprecht, I., Seymour, R. S. and Schultze-Motel, P., Direct and indirect calorimetry of thermogenic flowers of the sacred lotus, Nelumbo nucifera, Thermochim. Acta, 309 (1998), 5-16.

[81] Schultze-Motel, P., Heat loss and thermoregulation in a nest of the bumblebee Bombus Zapidariu, Thermochim. Acta, 193 (1991), 57-66.

[82] Birou, B. and von Stockar, U., Application of bench-scale calorimetry to chemostat cultures, Enzyme Microb. Technol., 11 (1989), 12-16.

[83] Duboc, P., Transient growth of Saccharomyces cerevisiae, A quantitative approach, PhD Thesis no. 1648, Swiss Federal Institute of Technology Lausanne (EPFL), 1997.

[84] von Stockar, U. and Birou B., The heat generated by yeast cultures with a mixed metabolism in the transition between respiration and fermentation, Biotechnol. Bioeng., 34 (1989), 86-101. 


\section{A Deriving heat balance equations for calorimetry from the first law}

Since calorimetric measuring devices are open systems often operating in transients as shown in Figure 2, it is most useful to reformulate the First Law $\mathrm{d} H=\delta Q+\delta W+V \mathrm{~d} p$ as follows:

$$
\frac{\mathrm{d} H}{\mathrm{~d} t}=\dot{Q}+\dot{W}+V \frac{\mathrm{d} p}{\mathrm{~d} t}+\sum_{e} \sum_{i} \dot{n}_{i, e} \cdot \bar{h}_{i, e},
$$

where the last term on the right hand represents the sum of the enthalpy associated with compounds entering or leaving the system (see Figure 2). $\dot{n}_{i, e}$ represents the molar flow rate of the $i$-th compound entering through the $e$-th exchange port (negative if leaving) and $\bar{h}_{i, e}$ denotes the partial molar enthalpy of $i$ at the conditions prevailing at the $e$-th exchange port. $V$ is the volume of the culture broth. According to the First Law of Thermodynamics, Eq. (A1) does not contain a source term.

$\mathrm{d} H / \mathrm{d} t$ may be expressed in terms of measurable variables by the following total differential:

$$
\frac{\mathrm{d} H}{\mathrm{~d} t}=C_{P} \cdot \frac{\mathrm{d} T}{\mathrm{~d} t}+\left(\frac{\partial H}{\partial p}\right)_{\mathrm{T}} \frac{\mathrm{d} p}{\mathrm{~d} t}+\sum_{i} \bar{h}_{i} \frac{\mathrm{d} n_{i}}{\mathrm{~d} t},
$$

where $\bar{h}_{i}$ represents the partial molar enthalpy of the $i$-th compound within the system. The rate of change of the number of moles of $i$ in the system $\mathrm{d} n_{i} / \mathrm{d} t$ may be calculated by the following molar balance:

$$
\frac{d n_{i}}{d t}=\sum_{e} \dot{n}_{i, e}+\sum_{j} v_{i, j} \cdot r_{j} \cdot V .
$$

In this equation, $r_{j}$ denotes the rate at which the $j$-th reaction proceeds (mol m $\mathrm{m}^{-3} \mathrm{~s}^{-1}$ ) and $v_{i, j}$ is the stoichiometric coefficient of the $i$-th compound in the $j$-th reaction. Expressing the rate of change of the enthalpy of the system $\mathrm{d} H / \mathrm{d} t$ in Eq. (A.1) by Eq. (A.2) and replacing $\mathrm{d} n_{i} / \mathrm{d} t$ by the molar balance Eq. (A.3) yields

$$
\begin{aligned}
C_{\mathrm{P}} \frac{\mathrm{d} T}{\mathrm{~d} t}= & \dot{Q}+\dot{W}+\left[V-\left(\frac{\partial H}{\partial p}\right)_{T, n_{i}}\right] \frac{\mathrm{d} p}{\mathrm{~d} t} \\
& +\sum_{e} \sum_{i}\left(\bar{h}_{i, e}-\bar{h}_{i}\right) \cdot \dot{n}_{i, e}-\sum_{j} \sum_{i} v_{i, j} \bar{h}_{i} \cdot r_{j} V .
\end{aligned}
$$


The heat capacity of the whole system $C_{\mathrm{P}}$ may be replaced by $\rho V \bar{c}_{\mathrm{P}}$, where $\bar{c}_{\mathrm{P}}$ denotes the heat capacity of $1 \mathrm{~kg}$ of aqueous broth. Treating the system as pseudo-homogeneous, the pressure dependence of its enthalpy may be replaced by the following exact thermodynamic relation:

$$
\left(\frac{\partial H}{\partial p}\right)_{T, n_{i}}=V-T\left(\frac{\partial V}{\partial T}\right)_{p, n_{i}} .
$$

Whence

$$
\left[V-\left(\frac{\partial H}{\partial p}\right)_{T, n_{i}}\right]=T\left(\frac{\partial V}{\partial T}\right)_{p, n_{i}} .
$$

The temperature expansion of the volume of aqueous liquids at constant pressure is however negligibly small, so that the whole term may be neglected for biotechnology. For the same reason, $\bar{h}_{i, e}-\bar{h}_{i}$ may be assumed to be pressure independent. Since both the culture broth and the entering and leaving streams are aqueous suspensions, the same heat capacity may be used for both. Hence,

$$
\bar{h}_{i, e}-\bar{h}_{i}=\bar{c}_{\mathrm{Pm}} \cdot\left(T_{e}-T\right),
$$

where $\mathrm{T}_{e}$ and $T$ stand for the temperature in the entering or leaving stream and in the culture broth, respectively, and $\overline{\mathrm{c}}_{\mathrm{Pm}}$ for the molar heat capacity of the aqueous suspension. The sum $\sum_{i} v_{i, j} \bar{h}_{i}$ is commonly designated by $\Delta_{r j} H$, the molar enthalpy change of the $\mathrm{j}$-th reaction (heat of reaction).

Introducing these simplifications into Eq. (A4) yields

$$
\rho V \bar{c}_{\mathrm{P}} \frac{\mathrm{d} T}{\mathrm{~d} t}=\dot{Q}+\dot{W}+\sum_{e} \bar{c}_{\mathrm{Pm}}\left(T_{e}-T\right) \cdot \dot{n}_{e}-\sum_{j} \Delta_{r j} H \cdot r_{j} V .
$$

Allowing for $\bar{c}_{\mathrm{Pm}} \cdot \dot{n}_{e}=\bar{c}_{\mathrm{P}} \cdot \rho \dot{V}_{e}$ yields Eq. (1) of the main body of the paper.

\section{B Several formal ways to write biosynthetic reactions $[3,9]$}

Macrochemical growth equations of the type shown in Eq. (2) may be split up formally into an energy-yielding (catabolic) and a biosynthetic (anabolic) reaction in many different ways. In the way of an example, 
consider the growth stoichiometry of yeast growing aerobically on a carbohydrate $[3,9]$ :

$$
\begin{aligned}
1.758 \mathrm{CH}_{2} \mathrm{O}+ & 0.695 \mathrm{O}_{2}+0.15 \mathrm{NH}_{3} \\
& \rightarrow \mathrm{CH}_{1.75} \mathrm{O}_{0.52} \mathrm{~N}_{0.15}+0.758 \mathrm{CO}_{2}+1.11 \mathrm{H}_{2} \mathrm{O} .
\end{aligned}
$$

The first chemical formula in this equation $\left(\mathrm{CH}_{2} \mathrm{O}\right)$ denotes a $\mathrm{C}$-mole of a carbohydrate such as $\mathrm{C}_{6} \mathrm{H}_{12} \mathrm{O}_{6}$, which is assumed to serve as energy source. The first formula on the right-hand side of Eq. (B1) stands for a C-mole of dry biomass. (The latter also contains between 5\% and $12 \%$ other elements, commonly denoted as "ash," which have been disregarded in the above representation).

Since no other products such as ethanol were released in the supernatant, growth was purely oxidative and the energy-yielding reactions (a) was respiration, which may be formalized as

$$
\mathrm{CH}_{2} \mathrm{O}+\mathrm{O}_{2} \rightarrow \mathrm{CO}_{2}+\mathrm{H}_{2} \mathrm{O}
$$

where $\mathrm{CH}_{2} \mathrm{O}$ denotes a $\mathrm{C}$-mole of carbohydrate. This equation is a specific example of reaction (a) presented in Eq. (16) in the main text.

However, as seen from comparing Figures 10 and 11, various variants may be imagined for the synthesis of $1 \mathrm{C}$-mol of dry biomass. Even the biosynthetic reaction shown in Figure 10 could be formalized in at least two different ways:

(i) Synthesis directly from the carbon and energy source by producing carbon dioxide:

$$
\begin{aligned}
& 1.065 \mathrm{CH}_{2} \mathrm{O}+0.15 \mathrm{NH}_{3} \\
& \quad \rightarrow \mathrm{CH}_{1.75} \mathrm{O}_{0.52} \mathrm{~N}_{0.15}+0.065 \mathrm{CO}_{2}+0.415 \mathrm{H}_{2} \mathrm{O}
\end{aligned}
$$

(ii) Synthesis directly from the carbon end energy source by producing oxygen:

$$
\begin{aligned}
\mathrm{CH}_{2} \mathrm{O}+ & 0.15 \mathrm{NH}_{3} \\
& \rightarrow \mathrm{CH}_{1.75} \mathrm{O}_{0.52} \mathrm{~N}_{0.15}+0.065 \mathrm{O}_{2}+0.35 \mathrm{H}_{2} \mathrm{O} .
\end{aligned}
$$

Figure 11 assumes that all the carbon and energy products are first catatabolized. Hence, 
(iii) Synthesis of fresh biomass from the products of catabolism:

$$
\begin{aligned}
\mathrm{CO}_{2}+0.15 \mathrm{NH}_{3}+0.65 & \mathrm{H}_{2} \mathrm{O} \\
& \rightarrow \mathrm{CH}_{1.75} \mathrm{O}_{0.52} \mathrm{~N}_{0.15}+1.065 \mathrm{O}_{2} .
\end{aligned}
$$

The three equations (B.3a)-(B.3c) are specific examples for reaction (b) presented in Eq. (16) of the main text.

Any one of them can be combined with the catabolic (B.2) reactions to yield the experimentally observed stoichiometry equation (B.1). This must be done in such a way as to obtain a stoichiometric coefficient of unity for the biomass and of 1.758 for the carbon and energy source. (Since this coefficient represents the inverse of the biomass yield $Y_{\mathrm{X} / \mathrm{S}}$, the latter amounts to $1 / 1.758=0.659)$. To this effect, one of the biosynthetic equations (B.3a)-(B.3c) must be added to the catabolic equation (B.2) multiplied with a factor of $\frac{1-Y_{\mathrm{SOX}}^{\mathrm{b}} Y_{\mathrm{X} / \mathrm{s}}}{Y_{\mathrm{X} / \mathrm{S}}}, \frac{1-Y_{\mathrm{X} / \mathrm{s}}}{Y_{\mathrm{X} / \mathrm{S}}}$, or $\frac{1}{Y_{\mathrm{X} / \mathrm{S}}}$ for cases (i), (ii), or (iii), respectively. ( $Y_{\text {SOX }}^{\mathrm{b}}$ is the stoichiometric coefficient for $\mathrm{CO}_{2}$ in Eq. (B.3a) and is equal to 0.065 in the above numerical case.) This procedure will always yield Eq. (B.1) shown above.

From Eq. (B.1), $\Delta_{r} G_{\mathrm{X}}^{0}$ and $\Delta_{r} H_{\mathrm{X}}^{0}$ may be computed by using Eq. (15) of the main text. However, it is simpler to calculate the Gibbs energy change $\Delta G_{\mathrm{a}}^{0}$ or the enthalpy change of the catabolic reaction directly from Eq. (B.2) and the Gibbs energy change $\Delta G_{\mathrm{b}}^{0}$ (or the enthalpy change) for biosynthesis from the appropriate biosynthetic stiochiometry (B.3a), (B.3b), or (B.3c). $\Delta_{r} G_{\mathrm{X}}^{0}$ and $\Delta_{r} H_{\mathrm{X}}^{0}$ are then obtained by using Eqs. (20a) and (20b), respectively. However, if the formal anabolic reaction was formulated according to case (i) or (ii), the factor $1 / Y_{\mathrm{X} / \mathrm{S}}$ in the first right-hand side term of Eq. (20) would have to be replaced by

$$
\frac{1-Y_{\mathrm{SOX}}^{\mathrm{b}} Y_{\mathrm{X} / \mathrm{S}}}{Y_{\mathrm{X} / \mathrm{S}}} \text { or } \frac{1-Y_{\mathrm{X} / \mathrm{S}}}{Y_{\mathrm{X} / \mathrm{S}}}
$$

for cases (i) and (ii), respectively.

Even with catabolic reactions other than respiration this computational procedure remains simple. Since the number of stoichiometric coefficients in the energy-yielding and biosynthetic reactions as formulated in Eqs. (16a) and (16b) matches the number of elements, they have no degree of freedom and they are readily equilibrated. (The general macrochemical equation shown in Eq. (19) has five coefficients, which are constrained by four elemental balances, thus leaving the biomass yield as the only degree of freedom). 
Received December 16, 2009; accepted February 2, 2010.

\section{Author information}

Urs von Stockar, Laboratoire de Génie Chimique et Biologique, ISIC - Station 6, Swiss Federal Institute of Technology, CH-1015 Lausanne, Switzerland.

E-mail: urs.vonstockar@epfl.ch 